\title{
Application of Machine Learning in Diagnosis of COVID-19 Through X-Ray and CT Images: A Scoping Review
}

\begin{abstract}
Hossein Mohammad-Rahimi ${ }^{1}$, Mohadeseh Nadimi ${ }^{2,3}$, Azadeh Ghalyanchi-Langeroudi ${ }^{2,3}$, Mohammad Taheri ${ }^{4 *}$ and Soudeh Ghafouri-Fard ${ }^{5 *}$

${ }^{1}$ Dental Research Center, Research Institute of Dental Sciences, Shahid Beheshti University of Medical Sciences, Tehran, Iran, ${ }^{2}$ Department of Medical Physics and Biomedical Engineering, Tehran University of Medical Sciences (TUMS), Tehran, Iran, ${ }^{3}$ Research Center for Biomedical Technologies and Robotics (RCBTR), Tehran, Iran, ${ }^{4}$ Urology and Nephrology Research Center, Shahid Beheshti University of Medical Sciences, Tehran, Iran, ${ }^{5}$ Department of Medical Genetics, Shahid Beheshti University of Medical Sciences, Tehran, Iran
\end{abstract}

Coronavirus disease, first detected in late 2019 (COVID-19), has spread fast throughout the world, leading to high mortality. This condition can be diagnosed using RT-PCR technique on nasopharyngeal and throat swabs with sensitivity values ranging from 30 to $70 \%$. However, chest CT scans and X-ray images have been reported to have sensitivity values of 98 and 69\%, respectively. The application of machine learning methods on CT and X-ray images has facilitated the accurate diagnosis of COVID-19. In this study, we reviewed studies which used machine and deep learning methods on chest $X$-ray images and CT scans for COVID-19 diagnosis and compared their performance. The accuracy of these methods ranged from $76 \%$ to more than $99 \%$, indicating the applicability of machine and deep learning methods in the clinical diagnosis of COVID-19.

Keywords: COVID-19, machine learning, detection, biomarker, X-ray image

\section{INTRODUCTION}

First identified in Wuhan, China, severe pneumonia caused by Severe Acute Respiratory Syndrome Coronavirus 2 (SARS-CoV-2) quickly spread all over the world. The resultant disorder was named coronavirus disease (COVID-19) $(1,2)$. COVID-19 has various clinical symptoms, including fever, cough, dyspnea, fatigue, myalgia, headache, and gastrointestinal complications (3-5). Diagnosis of COVID-19 infection through RT-PCR on nasopharyngeal and throat swab samples has been reported to yield positive results in $30-70 \%$ of cases $(6,7)$. On the other hand, chest CT scans and X-ray images have been reported to have sensitivity values of 98 and $69 \%$, respectively (7-9). The most typical radiological signs in these patients include multifocal and bilateral ground-glass opacities and consolidations, particularly in the peripheral and basal sites (10). However, interpretation of the results of these imaging techniques by expert radiologists might encounter some problems leading to reduced sensitivity (11). Artificial intelligence has recently gained the attention of both clinicians and researchers for the appropriate management of the COVID-19 pandemic (12). As an accurate method, artificial intelligence is able to identify abnormal patterns of CT and X-ray images. Using this method, it is possible to assess certain segment regions and take precise structures in chest CT images facilitating diagnostic purposes. Artificial intelligence methods have been shown to detect COVID-19 and distinguish this condition from other pulmonary disorders 
and community-acquired pneumonia (13). Both deep learning and machine learning approaches have been used to predict different aspects of the COVID-19 outbreak. Support vector and random forest are among the most applied machine learning methods, while Convolutional Neural Network (CNN), Long Short-Term Memory (LSTM), Generative Adversarial Networks (GAN), and Residual Neural network are among the deep learning methods used in this regard (14). In this study, we reviewed studies which used machine and deep learning methods on chest X-ray images and CT scans for the purpose of COVID19 diagnosis and compared their performance.

\section{METHODS}

\section{Search Strategy}

The research question was: "What are the applications of machine learning techniques and their performances in COVID19 diagnosis using X-ray images?". The search of the present review was based on the PICO elements, which were as follows:

- P (Problem/Patient/Population): Patients' CT scans and Chest X-rays.

- I (Intervention/Indicator): Machine/deep learning models for diagnosis of Covid-19 patients

- C (Comparison): Ground truth or reference standards

- O (Outcome): Performance measurements including accuracy, AUC score, sensitivity, and specificity.

In other words, we were looking for publications that evaluated the performance of any machine learning or deep learning approaches based on inclusion and exclusion criteria. Studies that used other types of medical image modalities (e.g., ultrasound images) were excluded. An electronic search was conducted on PubMed, Google Scholar, Scopus, Embase, arXiv, and medRxiv for finding the relevant literature. Duplicate studies were removed. Studies that were cited within the retrieved papers were reviewed for finding missing studies. For identifying proper journal papers and conference proceedings, investigators screened the title and abstracts based on inclusion and exclusion criteria independently. Finally, considering the inclusion and exclusion criteria, investigators identified the eligible publications in this stage independently.

\section{Inclusion Criteria}

The following inclusion criteria were used in the selection of the articles: (1) Studies that applied machine learning or deep learning algorithms, (2) Studies that evaluated the measurement of model outcomes in comparison with ground truth or gold standards, and (3) Studies that used algorithms to analyze radiographic images (CT scan, Chest X-ray, etc.).

\section{Exclusion Criteria}

The following studies were excluded: (1) Studies that used any machine learning or deep learning approaches for problems not directly related to the COVID-19 imaging, (2) Studies that used other artificial intelligence techniques or classic computer vision approaches, (3) Studies that did not provide a clear explanation of the machine learning or deep learning model that was used to solve their problem, and (4) Review studies. The latter were excluded as we did not aim to review the data on an original level without any second-hand interpretations (summation, inferences, etc.).

Figure 1 shows the flowchart of the study design.

\section{RESULTS}

We obtained 105 studies that used machine or deep learning methods to assess chest images of COVID-19 patients. These studies have used different analytical methods. For instance, Ardakani et al. (15) have assessed radiological features of CT images obtained from patients with COVID-19 and nonCOVID-19 pneumonia. They used decision tree, K-nearest neighbor, naïve Bayes, support vector machine, and ensemble classifiers to find the computer-aided diagnosis system with the best performance in distinguishing COVID-19 patients from non-COVID-19 pneumonia. They reported that site and distribution of pulmonary involvement, the quantity of the pulmonary lesions, ground-glass opacity, and crazy-paving as the most important characteristics for differentiation of these two sets of patients. Their computer-aided diagnosis method yielded the accuracy of $91.94 \%$, using an ensemble (COVIDiag) classifier. Alazab et al. (16) have developed an artificialintelligence method based on a deep CNN to evaluate chest Xray images and detection of COVID-19 patients. Their method yielded an F-measure ranging from 95 to $99 \%$. Notably, three predicting strategies could forecast the numbers of COVID-19 confirmations, recoveries, and mortalities over the upcoming week. The average accuracy of the prediction models were 94.80 and $88.43 \%$ in two different countries. Albahli has applied deep learning-based models on CT images of COVID-19 patients. He has demonstrated a high performance of a Deep Neural Network model in detecting COVID-19 patients and has offered an efficient assessment of chest-related disorders according to age and sex. His proposed model has yielded $89 \%$ accuracy in terms of GAN-based synthetic data (17). Automatic detection of COVID-19 based on X-ray images has been executed through the application of three deep learning models, including Inception ResNetV2, InceptionNetV3, and NASNetLarge. The best results have been obtained from InceptionNetV3, which yielded the accuracy levels of 98.63 and $99.02 \%$ with and without application of data augmentation in model training, respectively (18). Alsharman et al. (19) have used the CNN method to detect COVID-19 based on chest CT images in the early stages of disease course. Authors have reported high accuracy of GoogleNet CNN architecture for diagnosis of COVID-19. Altan et al. (20) have used a hybrid model comprising two-dimensional curvelet transformation, chaotic salp swarm algorithm, and deep learning methods for distinguishing COVID-19 from other pneumonia cases. Application of their proposed model on chest X-ray images has led to accurate diagnosis of COVID-19 patients (Accuracy $=99.69 \%$, Sensitivity $=99.44 \%$ and Specificity $=$ 99.81\%). Apostolopoulos et al. (21) have used a certain CNN strategy, namely MobileNet on X-Ray images of COVID-19 patients. This method has yielded more than $99 \%$ accuracy 


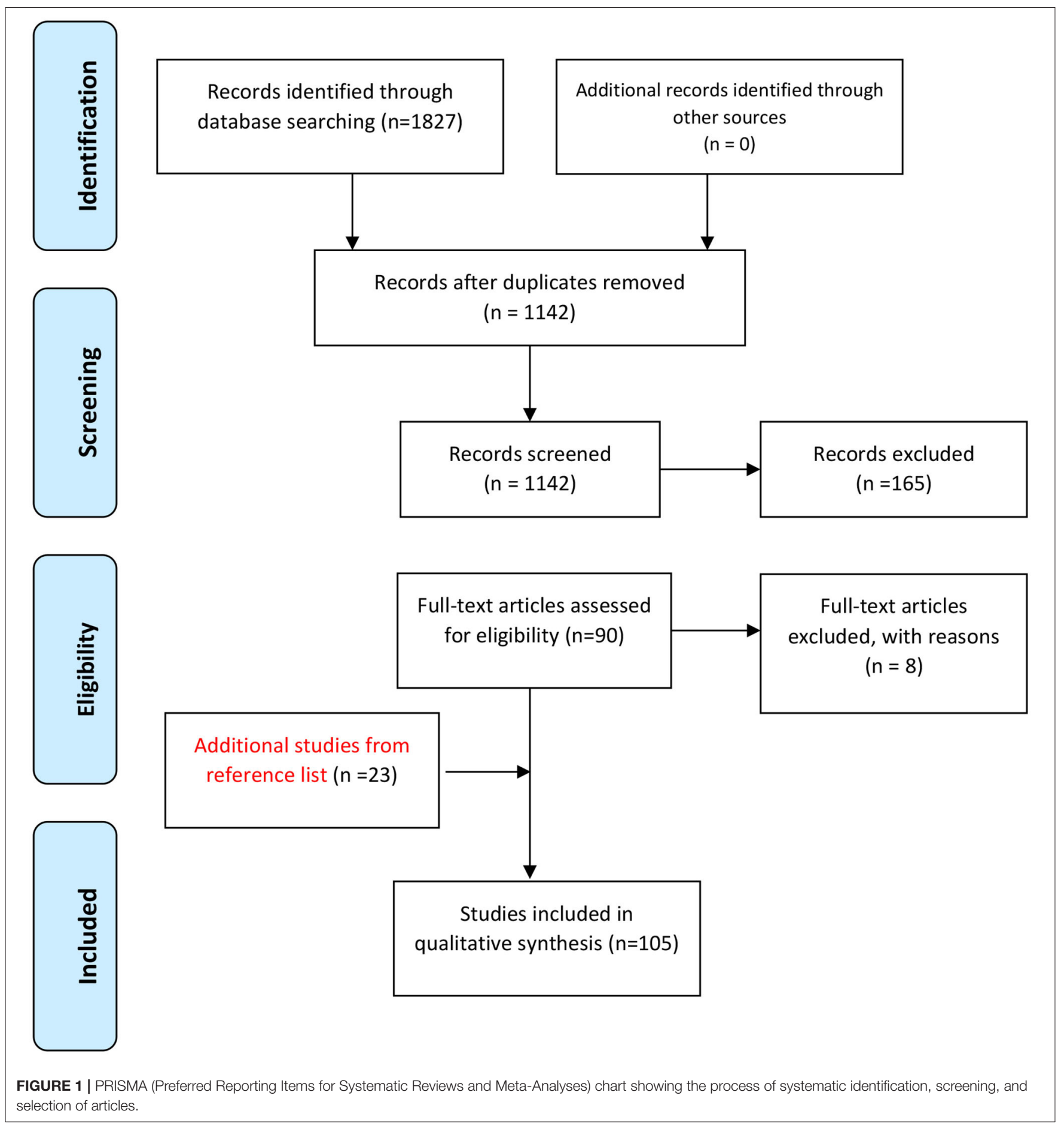

in the diagnosis of COVID-19. In another study, Ardakani et al. (22) used $10 \mathrm{CNN}$ strategies, namely AlexNet, VGG16, VGG-19, SqueezeNet, GoogleNet, MobileNet-V2, ResNet18, ResNet-50, ResNet-101, and Xception, to differentiate COVID-19 cases from non-COVID-19 patients. They have demonstrated the best diagnostic values for ResNet-101 and Xception, both of them having area under curve (AUC) values higher than 0.99 which is superior to the performance of the radiologist. Das et al. (23) have used the CNN model Truncated InceptionNet to diagnose COVID-19 from other non-COVID and/or healthy cases based on chest X-ray. Their suggested model yielded AUC of 1.0 in distinguishing COVID-19 patients from combined Pneumonia and healthy subjects. Tables 1, 2 summarize the features of studies which adopted machine learning methods in CT images and chest X-ray of COVID-19 patients, respectively. 
TABLE 1 | Characteristics of papers that used CT images or a combination of X-ray and CT images

\begin{tabular}{|c|c|c|c|c|c|c|c|c|c|}
\hline \multirow[t]{2}{*}{ Author, year } & \multirow[t]{2}{*}{ Data source } & \multirow{2}{*}{$\begin{array}{l}\text { Data structure and } \\
\text { size }\end{array}$} & \multirow[t]{2}{*}{ Data preprocessing } & \multirow{2}{*}{$\begin{array}{l}\text { Best model } \\
\text { structure(s) }\end{array}$} & \multicolumn{4}{|c|}{ Performance measurements (on the best model) } & \multirow[t]{2}{*}{ References } \\
\hline & & & & & Accuracy & AUC score & Sensitivity & Specificity & \\
\hline $\begin{array}{l}\text { Abbasian et al. } \\
\text { (2020) }\end{array}$ & $\begin{array}{l}\text { Iran University of } \\
\text { Medical Sciences } \\
\text { (IUMS) }\end{array}$ & $\begin{array}{l}306 \text { COVID-19 } \\
\text { patients; } \\
306 \text { COVID-19 } \\
\text { pneumonia (CT images) }\end{array}$ & $\begin{array}{l}\text { Extracting } 20 \text { features of CT } \\
\text { images }\end{array}$ & Ensemble & $91.94 \%$ & 0.965 & $93.54 \%$ & $90.32 \%$ & (15) \\
\hline $\begin{array}{l}\text { Alsharman et al. } \\
\text { (2020) }\end{array}$ & "COVID-CT-dataset" & CT images & $\begin{array}{l}\text { Binarization (the separation of } \\
\text { the object and background is } \\
\text { known as Binarization); } \\
\text { Converting input image from 2D } \\
\text { Grayscale to 3D Color }\end{array}$ & GoogleNet CNN & $82.14 \%$ & & & & (19) \\
\hline $\begin{array}{l}\text { Ardakani et al. } \\
\text { (2020) }\end{array}$ & Private dataset & $\begin{array}{l}108 \text { COVID-19 } \\
\text { patients; } \\
86 \text { viral pneumonia } \\
\text { diseases (CT images) }\end{array}$ & $\begin{array}{l}\text { Converted to the gray-scale } \\
\text { Cropped and resized to } 60 \text { * } 60 \\
\text { pixels }\end{array}$ & $\begin{array}{l}\text { ResNet-101 } \\
\text { Xception }\end{array}$ & $\begin{array}{l}\text { Resnet: } \\
\text { 99.51\% } \\
\text { Xception: } \\
99.02 \% \\
\text { (compared to } \\
86.7 \% \\
\text { in human) }\end{array}$ & $\begin{array}{l}\text { Resnet: } 0.994 \\
\text { Xception: } \\
0.994 \% \\
\text { (compared to } \\
0.873 \\
\text { in human) }\end{array}$ & $\begin{array}{l}\text { Resnet: } \\
100 \% \\
\text { Xception: } \\
98.04 \% \\
\text { (compared to } \\
89.21 \% \\
\text { in human) }\end{array}$ & $\begin{array}{l}\text { Resnet: } \\
\text { 99.02\% } \\
\text { Xception: } \\
100 \% \\
\text { (compared to } \\
83.33 \% \\
\text { in human) }\end{array}$ & (22) \\
\hline $\begin{array}{l}\text { Aswathy et al. } \\
\text { (2020) }\end{array}$ & $\begin{array}{l}\text { "National Cancer } \\
\text { Institute and the } \\
\text { Cancer Image Archive" }\end{array}$ & $\begin{array}{l}1,763 \text { normal patients; } \\
63 \text { pneumonia patients }\end{array}$ & $\begin{array}{l}\text { Thresholding; } \\
\text { Texture-based feature } \\
\text { extractionwith a wrapper }\end{array}$ & CNN & $99 \%$ & - & - & - & (24) \\
\hline Bai et al. (2020) & Private dataset & $\begin{array}{l}521 \text { COVID-19 } \\
\text { patients; } \\
665 \text { other pulmonary } \\
\text { diseases (CT images) }\end{array}$ & $\begin{array}{l}\text { Lung segmentation; } \\
\text { Generate an 8-bit image for each } \\
\text { axial slice by applying Lung } \\
\text { windowing to the } \\
\text { Hounsfield units }\end{array}$ & EfficientNet B4 & $\begin{array}{l}96 \% \\
\text { (compared to } \\
85 \% \text { in } \\
\text { human) }\end{array}$ & 0.95 & $\begin{array}{l}95 \% \\
\text { (compared to } \\
79 \% \text { in } \\
\text { human) }\end{array}$ & $\begin{array}{l}96 \% \\
\text { (compared to } \\
88 \% \text { in } \\
\text { human) }\end{array}$ & (11) \\
\hline Bridge et al. (2020) & $\begin{array}{l}\text { "Toy dataset;" } \\
\text { "Italian Society of } \\
\text { Radiology;" } \\
\text { "Shenzhen Hospital } \\
\text { X-Ray dataset;" } \\
\text { "ChestX-Ray8;" } \\
\text { "COVID-CT-Dataset" }\end{array}$ & $\begin{array}{l}129 \text { COVID-19 } \\
\text { patients; } \\
62,267 \text { normal } \\
\text { patients; } \\
5,689 \text { pneumonia } \\
\text { patients (X-ray images) } \\
30 \text { COVID-19 patients; } \\
\text { 1,919 normal patients } \\
\text { (CT images) }\end{array}$ & $\begin{array}{l}\text { Using the GEV activation } \\
\text { function for unbalanced data }\end{array}$ & Inception V3 & $100 \%$ & - & $100 \%$ & $100 \%$ & (25) \\
\hline Butt et al. (2020) & Not mentioned & $\begin{array}{l}219 \text { images from } 110 \\
\text { COVID-19 patients; } \\
399 \text { normal patients } \\
\text { (CT images) }\end{array}$ & $\begin{array}{l}\text { Image processing method base } \\
\text { on } \mathrm{HU} \text { values }\end{array}$ & 3D CNN & - & 0.996 & $98.2 \%$ & $92.2 \%$ & (26) \\
\hline
\end{tabular}


TABLE 1 | Continued

\begin{tabular}{|c|c|c|c|c|c|c|c|c|c|}
\hline \multirow[t]{2}{*}{ Author, year } & \multirow[t]{2}{*}{ Data source } & \multirow{2}{*}{$\begin{array}{l}\text { Data structure and } \\
\text { size }\end{array}$} & \multirow[t]{2}{*}{ Data preprocessing } & \multirow{2}{*}{$\begin{array}{l}\text { Best model } \\
\text { structure(s) }\end{array}$} & \multicolumn{4}{|c|}{ Performance measurements (on the best model) } & \multirow[t]{2}{*}{ References } \\
\hline & & & & & Accuracy & AUC score & Sensitivity & Specificity & \\
\hline Dey et al. (2020) & $\begin{array}{l}\text { "COVID-19 CT } \\
\text { segmentation dataset;" } \\
\text { "Chest } \\
\text { X-rays (Radiopaedia)" }\end{array}$ & $\begin{array}{l}200 \text { COVID-19 } \\
\text { patients; } \\
200 \text { normal patients } \\
\text { (grayscale lung } \\
\text { CTI images) }\end{array}$ & $\begin{array}{l}\text { Segmenting lung area related to } \\
\text { pneumonia infection; } \\
\text { Extracting CWT, DWT, EWT } \\
\text { features from original image and } \\
\text { Haralick, Hu moments from } \\
\text { binary segmented area } \\
\text { Feature selection based on } \\
\text { statistical tests }\end{array}$ & KNN & $87.75 \%$ & - & $89.00 \%$ & $86.50 \%$ & (27) \\
\hline $\begin{array}{l}\text { El Asnaoui et al. } \\
\text { (2020) }\end{array}$ & $\begin{array}{l}\text { COVID-19 X-ray image } \\
\text { database developed by } \\
\text { Cohen JP; } \\
\text { Kermany et al. (28) }\end{array}$ & $\begin{array}{l}\text { 2,780 Bacterial } \\
\text { pneumonia patients; } \\
\text { 1,493 Coronavirus } \\
\text { patients; } \\
\text { 231 COVID-19 } \\
\text { patients; } \\
\text { 1,583 normal patients } \\
\text { (X-ray and CT images) }\end{array}$ & $\begin{array}{l}\text { Intensity Normalization; } \\
\text { Contrast Limited Adaptive } \\
\text { Histogram Equalization }\end{array}$ & $\begin{array}{l}\text { Inception } \\
\text { ResNetV2; } \\
\text { Densnet201 }\end{array}$ & $\begin{array}{l}\text { Inception- } \\
\text { ResNetV2: } \\
\text { 92.18\% } \\
\text { Densnet201: } \\
\text { 88.09\% }\end{array}$ & $\begin{array}{l}\text { Inception- } \\
\text { ResNetV2: } \\
0.920 \\
\text { Densnet201: } \\
0.879\end{array}$ & $\begin{array}{l}\text { Inception- } \\
\text { ResNetV2: } \\
\text { 92.11\% } \\
\text { Densnet201: } \\
87.99 \%\end{array}$ & $\begin{array}{l}\text { Inception- } \\
\text { ResNetV2: } \\
\text { 96.6\% } \\
\text { Densnet201: } \\
\text { 94.00\% }\end{array}$ & (29) \\
\hline Han et al. (2020) & $\begin{array}{l}\text { "COVID-19 hospitals in } \\
\text { Shandong Province" }\end{array}$ & $\begin{array}{l}79 \text { COVID-19 patients; } \\
100 \text { pneumonia } \\
\text { patients; } \\
130 \text { normal patients } \\
\text { (CT images) }\end{array}$ & Data augmentation & AD3D-MIL & $97.9 \%$ & 0.99 & $97.9 \%$ & $97.9 \%$ & (30) \\
\hline $\begin{array}{l}\text { Harmon et al. } \\
\text { (2020) }\end{array}$ & Private dataset & $\begin{array}{l}386 \text { COVID-19 } \\
\text { patients; } \\
1,011 \text { negative } \\
\text { COVID-19 patients } \\
\text { (CT images) }\end{array}$ & $\begin{array}{l}\text { Lung segmentation; clipping } \\
\text { images to HU range (-1,000, } \\
500) ; \\
\text { Data augmentation (flipping, } \\
\text { rotation, image intensity and } \\
\text { contrast adjustment, adding } \\
\text { random Gaussian noise); }\end{array}$ & $\begin{array}{l}\text { Hybrid 3D based } \\
\text { on Densnet-121 }\end{array}$ & $90.8 \%$ & - & $84 \%$ & $93 \%$ & (31) \\
\hline Hasan et al. (2020) & $\begin{array}{l}\text { "Radiopaedia and the } \\
\text { cancer imaging archive } \\
\text { websites" }\end{array}$ & $\begin{array}{l}118 \text { COVID-19 } \\
\text { patients; } 96 \text { pneumonia } \\
\text { patients; } \\
107 \text { normal patients } \\
\text { (CT images) }\end{array}$ & $\begin{array}{l}\text { Histogram } \\
\text { Thresholding; } \\
\text { Dilation; } \\
\text { Hole Filling }\end{array}$ & LSTM & $99.68 \%$ & - & $100 \%$ & - & (32) \\
\hline
\end{tabular}


TABLE 1 | Continued

\begin{tabular}{|c|c|c|c|c|c|c|c|c|c|}
\hline \multirow[t]{2}{*}{ Author, year } & \multirow[t]{2}{*}{ Data source } & \multirow{2}{*}{$\begin{array}{l}\text { Data structure and } \\
\text { size }\end{array}$} & \multirow[t]{2}{*}{ Data preprocessing } & \multirow{2}{*}{$\begin{array}{l}\text { Best model } \\
\text { structure(s) }\end{array}$} & \multicolumn{4}{|c|}{ Performance measurements (on the best model) } & \multirow[t]{2}{*}{ References } \\
\hline & & & & & Accuracy & AUC score & Sensitivity & Specificity & \\
\hline Hu et al. (2020) & $\begin{array}{l}\text { "Hospital of Wuhan } \\
\text { Red Cross Society;" } \\
\text { "Shenzhen Hospital;" } \\
\text { "TCIA dataset;" } \\
\text { "Cancer Centre Archive } \\
\text { (TCIA) Public Access;" } \\
\text { "MD Anderson Cancer } \\
\text { Centre;" } \\
\text { "Memorial } \\
\text { Sloan-Kettering Cancer } \\
\text { Center;" } \\
\text { "MAASTRO clinic" }\end{array}$ & $\begin{array}{l}150 \text { COVID-19 } \\
\text { patients; } \\
150 \text { pneumonia } \\
\text { patients; } \\
150 \text { normal patients } \\
\text { (CT images) }\end{array}$ & Data augmentation & CNN & $96.2 \%$ & 0.970 & $94.5 \%$ & $95.3 \%$ & (33) \\
\hline $\begin{array}{l}\text { Jaiswal et al. } \\
(2020)\end{array}$ & $\begin{array}{l}\text { "The SARS-CoV-2 CT } \\
\text { scan dataset" }\end{array}$ & $\begin{array}{l}1,262 \text { COVID-19 } \\
\text { patients; } 1,230 \\
\text { non-COVID-19 patients } \\
\text { (CT images) }\end{array}$ & $\begin{array}{l}\text { Data augmentation (rotation up } \\
\text { to } 15, \text { slant-angle of } 0.2 \text {, } \\
\text { horizontal flipping, filling new } \\
\text { pixels as "nearest" for better } \\
\text { robustness) }\end{array}$ & DenseNet201 & $96.25 \%$ & 0.97 & $96.29 \%$ & $96.21 \%$ & (34) \\
\hline Kang et al. (2020) & $\begin{array}{l}\text { "Tongji Hospital of } \\
\text { Huazhong University of } \\
\text { Science } \\
\text { and Technology;" } \\
\text { "China-Japan Union } \\
\text { Hospital of Jilin } \\
\text { University;" } \\
\text { "Ruijin Hospital } \\
\text { ofShanghai Jiao } \\
\text { Tong University" }\end{array}$ & $\begin{array}{l}\text { 1,495 COVID-19 } \\
\text { patients; } \\
1,027 \\
\text { community-acquired } \\
\text { pneumonia (CAP) } \\
\text { patients (CT images) }\end{array}$ & $\begin{array}{l}\text { Normalization; } \\
\text { Standardization }\end{array}$ & NN & $93.90 \%$ & - & $94.60 \%$ & $91.70 \%$ & (35) \\
\hline $\begin{array}{l}\text { Lessmann et al. } \\
\text { (2020) }\end{array}$ & $\begin{array}{l}\text { "Emergency wards of } \\
\text { an Academic center } \\
\text { and teaching hospital in } \\
\text { the Netherlands in } \\
\text { March and April 2020" }\end{array}$ & $\begin{array}{l}237 \text { COVID-19 } \\
\text { patients; } \\
606 \text { normal patients } \\
\text { (CT images) }\end{array}$ & $\begin{array}{l}\text { Resampling; } \\
\text { Normalization }\end{array}$ & CORADS-Al & - & 0.95 & $85.7 \%$ & $89.8 \%$ & (36) \\
\hline Li et al. (2020) & Private & $\begin{array}{l}\text { 1,296 COVID-19 } \\
\text { patients; } \\
\text { 1,325-patients; } \\
1,735 \\
\text { community-acquired } \\
\text { (CT images) }\end{array}$ & Segmenting lung area with U-net & $\begin{array}{l}\text { COVNet } \\
\text { (ResNet-50) }\end{array}$ & - & 0.96 & $90 \%$ & $96 \%$ & (13) \\
\hline
\end{tabular}


TABLE 1 | Continued

\begin{tabular}{|c|c|c|c|c|c|c|c|c|c|}
\hline \multirow[t]{2}{*}{ Author, year } & \multirow[t]{2}{*}{ Data source } & \multirow{2}{*}{$\begin{array}{l}\text { Data structure and } \\
\text { size }\end{array}$} & \multirow[t]{2}{*}{ Data preprocessing } & \multirow{2}{*}{$\begin{array}{l}\text { Best model } \\
\text { structure(s) }\end{array}$} & \multicolumn{4}{|c|}{ Performance measurements (on the best model) } & \multirow[t]{2}{*}{ References } \\
\hline & & & & & Accuracy & AUC score & Sensitivity & Specificity & \\
\hline Li et al. (2020) & $\begin{array}{l}\text { More than } 10 \text { medical } \\
\text { centers between Nov. } \\
11 \text { th, } 2010 \text { and Feb. } \\
\text { 9th, } 2020\end{array}$ & $\begin{array}{l}305 \text { images from } 251 \\
\text { COVID-19 patients; } \\
872 \text { images from } 869 \\
\text { pneumonia patients; } \\
1,498 \text { images from } \\
1,475 \text { non-pneumonia } \\
\text { patients (CT images) }\end{array}$ & $\begin{array}{l}\text { DL-based algorithm } \\
\text { Image processing method base } \\
\text { on HU values; } \\
\text { Data augmentation }\end{array}$ & 3D ResNet-18 & \multicolumn{4}{|c|}{ Recall $=88 \%$ Precision $=89.6 \%$ F1 score $=87.8 \%$} & (37) \\
\hline Liu et al. (2020) & Private & $\begin{array}{l}73 \text { COVID-19 patients; } \\
27 \text { general pneumonia } \\
\text { patients (CT images) }\end{array}$ & $\begin{array}{l}\text { ROI delineation based on } \\
\text { ground-glass opacities (GGOs); } \\
13 \text { gray level co-occurrence } \\
\text { matrix (GLCM) features, } 15 \text { gray } \\
\text { level-gradient co-occurrence } \\
\text { matrix (GLGCM) features, and six } \\
\text { histogram features were } \\
\text { extracted; } \\
\text { Feature selection by ReliefF; }\end{array}$ & $\begin{array}{l}\text { An ensemble of } \\
\text { bagged tree (EBT) }\end{array}$ & $94.16 \%$ & 0.99 & $88.62 \%$ & $100 \%$ & (38) \\
\hline Mei et al. (2020) & Private & $\begin{array}{l}419 \text { COVID-19 patients } \\
486 \text { non-COVID-19 } \\
\text { patients (CT images) }\end{array}$ & $\begin{array}{l}\text { Selecting pertinent slices by } \\
\text { image segmentation to detect } \\
\text { parenchymal tissue; } \\
\text { Segmenting lung in CT images; }\end{array}$ & ResNet-18 & $79.6 \%$ & 0.86 & $83.6 \%$ & $75.9 \%$ & (39) \\
\hline $\begin{array}{l}\text { Panwar et al. } \\
\text { (2020) }\end{array}$ & $\begin{array}{l}\text { "COVID-chest X-ray;" } \\
\text { "SARS-COV-2 } \\
\text { CT-scan;" } \\
\text { "Chest X-Ray } \\
\text { Images (Pneumonia);" }\end{array}$ & $\begin{array}{l}206 \text { COVID-19 } \\
\text { patients; } \\
364 \text { Pneumonia } \\
\text { patients (X-ray and } \\
\text { CT images) }\end{array}$ & - & VGG-19 & $\begin{array}{l}95.61 \% \\
\text { (COVID-19 } \\
\text { vs. } \\
\text { Pneumonia) }\end{array}$ & - & $\begin{array}{l}96.55 \% \\
\text { (COVID-19 } \\
\text { vs. } \\
\text { Pneumonia) }\end{array}$ & $\begin{array}{l}95.29 \% \\
\text { (COVID-19 } \\
\text { vs. } \\
\text { Pneumonia) }\end{array}$ & (40) \\
\hline $\begin{array}{l}\text { Pathak et al. } \\
(2020)\end{array}$ & $\begin{array}{l}2 \text { different COVID-19 } \\
\text { datasets of chest-CT } \\
\text { images }\end{array}$ & CT images & - & $\begin{array}{l}\text { Deep bidirectional } \\
\text { long short-term } \\
\text { memory network } \\
\text { with mixture } \\
\text { density network } \\
\text { (DBM) }\end{array}$ & $\begin{array}{l}96.19 \% \\
\text { (multi-class) }\end{array}$ & $\begin{array}{l}0.96 \\
\text { (multi-class) }\end{array}$ & $\begin{array}{l}96.22 \% \\
\text { (multi-class) }\end{array}$ & $\begin{array}{l}96.16 \% \\
\text { (multi-class) }\end{array}$ & (41) \\
\hline $\begin{array}{l}\text { Pathak et al. } \\
(2020)\end{array}$ & $\begin{array}{l}\text { "COVID-19 open } \\
\text { datasets of chest CT } \\
\text { images" }\end{array}$ & $\begin{array}{l}413 \text { COVID-19 } \\
\text { patients; } \\
439 \text { normal or } \\
\text { pneumonia infected } \\
\text { patients (CT images) }\end{array}$ & - & ResNet-50 & $93.01 \%$ & - & $91.45 \%$ & $94.77 \%$ & (41) \\
\hline Peng et al. (2020) & Collected from PMC & $\begin{array}{l}606 \text { COVID-19 } \\
\text { patients; } \\
222 \text { Influenza; } \\
397 \text { Normal or other } \\
\text { disease patients } \\
\text { (CT images) }\end{array}$ & - & DenseNet121 & - & 0.87 & $72.3 \%$ & $85.2 \%$ & (42) \\
\hline
\end{tabular}


TABLE 1 | Continued

\begin{tabular}{|c|c|c|c|c|c|c|c|c|c|}
\hline \multirow[t]{2}{*}{ Author, year } & \multirow[t]{2}{*}{ Data source } & \multirow{2}{*}{$\begin{array}{l}\text { Data structure and } \\
\text { size }\end{array}$} & \multirow[t]{2}{*}{ Data preprocessing } & \multirow{2}{*}{$\begin{array}{l}\text { Best model } \\
\text { structure(s) }\end{array}$} & \multicolumn{4}{|c|}{ Performance measurements (on the best model) } & \multirow[t]{2}{*}{ References } \\
\hline & & & & & Accuracy & AUC score & Sensitivity & Specificity & \\
\hline Pu et al. (2020) & Private & $\begin{array}{l}498 \text { COVID-19 } \\
\text { patients; } \\
497 \\
\text { community-acquired } \\
\text { pneumonia (CAP) } \\
\text { (CT images) }\end{array}$ & $\begin{array}{l}\text { Data augmentation [rotation, } \\
\text { translation, vertical/horizontal } \\
\text { flips, Hounsfield Unit (HU) shift, } \\
\text { smoothing (blurring) operation, } \\
\text { Gaussian noise] }\end{array}$ & 3D CNNs & $99 \%$ & 0.7 & - & - & (43) \\
\hline $\begin{array}{l}\text { Raajan et al. } \\
(2020)\end{array}$ & $\begin{array}{l}\text { X-ray images on public } \\
\text { medical Github } \\
\text { repositories; } \\
\text { Kaggle chest } \\
\text { X-ray database }\end{array}$ & $\begin{array}{l}349 \text { images from } 216 \\
\text { COVID-19 patients; } \\
1,341 \text { Normal patients } \\
\text { (CT images) }\end{array}$ & Normalization & ResNet-16 & $95.09 \%$ & - & $100 \%$ & $81.89 \%$ & (44) \\
\hline $\begin{array}{l}\text { Rajaraman et al. } \\
(2020)\end{array}$ & $\begin{array}{l}\text { "Pediatric CXR } \\
\text { dataset;" } \\
\text { "RSNA CXR dataset;" } \\
\text { "Twitter COVID-19 CXR } \\
\text { dataset;" } \\
\text { "Montreal COVID-19 } \\
\text { CXR dataset" }\end{array}$ & $\begin{array}{l}313 \text { COVID-19 } \\
\text { patients; } \\
7,595 \text { pneumonia of } \\
\text { unknown type patients; } \\
\text { 2,780 bacterial } \\
\text { pneumonia; } \\
\text { 7,595 Normal patients } \\
\text { (X-ray images) }\end{array}$ & $\begin{array}{l}\text { Median filtering; } \\
\text { Normalization; } \\
\text { Standardization }\end{array}$ & Inception-V3 & $99.01 \%$ & 0.997 & $98.4 \%$ & - & (45) \\
\hline $\begin{array}{l}\text { Sakagianni et al. } \\
(2020)\end{array}$ & $\begin{array}{l}\text { COVID-19 articles on } \\
\text { medRxiv and bioRxiv }\end{array}$ & $\begin{array}{l}349 \text { COVID-19 } \\
\text { patients; } \\
397 \text { non-COVID-19 } \\
\text { patients (CT images) }\end{array}$ & - & $\begin{array}{l}\text { AutoML Cloud } \\
\text { Vision }\end{array}$ & - & 0.94 & $88.31 \%$ & - & (46) \\
\hline Sharma (2020) & $\begin{array}{l}\text { Dataset from Italian } \\
\text { Society of Medical and } \\
\text { Interventional Radiology; } \\
\text { COVID-CT available in } \\
\text { GitHub; } \\
\text { Dataset from hospitals } \\
\text { in Moscow, Russia; } \\
\text { Dataset from SAL } \\
\text { Hospital, } \\
\text { Ahmedabad, India; }\end{array}$ & $\begin{array}{l}800 \text { COVID-19 } \\
\text { patients; } \\
600 \text { Viral Pneumonia; } \\
800 \text { normal patients } \\
\text { (CT images) } \\
\end{array}$ & $\begin{array}{l}\text { Ground-glass opacities (GGO), } \\
\text { consolidation and pleural } \\
\text { effusion are the features }\end{array}$ & ResNet & $91 \%$ & - & $92.1 \%$ & $90.29 \%$ & $(47)$ \\
\hline Singh et al. (2020) & Not mentioned & CT images & - & $\begin{array}{l}\text { Multi-objective } \\
\text { differential } \\
\text { evolution (MODE) } \\
\text { based CNN }\end{array}$ & $90.22 \%$ & - & $91.17 \%$ & $89.23 \%$ & (48) \\
\hline Song et al. (2020) & $\begin{array}{l}\text { Private (two hospitals in } \\
\text { China); }\end{array}$ & $\begin{array}{l}98 \text { COVID-19 patients; } \\
103 \text { non-COVID-19 } \\
\text { pneumonia (CT images) }\end{array}$ & - & BigBiGAN & - & 0.972 & $92 \%$ & $91 \%$ & (49) \\
\hline
\end{tabular}


TABLE 1 | Continued

\begin{tabular}{|c|c|c|c|c|c|c|c|c|c|}
\hline \multirow[t]{2}{*}{ Author, year } & \multirow[t]{2}{*}{ Data source } & \multirow{2}{*}{$\begin{array}{l}\text { Data structure and } \\
\text { size }\end{array}$} & \multirow[t]{2}{*}{ Data preprocessing } & \multirow{2}{*}{$\begin{array}{l}\text { Best model } \\
\text { structure(s) }\end{array}$} & \multicolumn{4}{|c|}{ Performance measurements (on the best model) } & \multirow[t]{2}{*}{ References } \\
\hline & & & & & Accuracy & AUC score & Sensitivity & Specificity & \\
\hline Wang et al. (2020) & Private & $\begin{array}{l}\text { 1,315 COVID-19 } \\
\text { patients; } \\
\text { 2,406 ILD patients; } \\
936 \text { Normal patients } \\
\text { (CT images) }\end{array}$ & $\begin{array}{l}\text { Lobe Segmentation by 3D-Unet; } \\
\text { Converting CT numbers } \\
\text { to grayscale }\end{array}$ & PA-66 model & $93.3 \%$ & 0.973 & $97.6 \%$ & - & (50) \\
\hline Wang et al. (2020) & $\begin{array}{l}\text { COVID-19 dataset } \\
\text { (private); } \\
\text { CT-epidermal growth } \\
\text { factor receptor } \\
\text { (CT-EGFR) } \\
\text { dataset (private); }\end{array}$ & $\begin{array}{l}754 \text { COVID-19 } \\
\text { patients; } \\
271 \text { bacterial } \\
\text { pneumonia } \\
29 \text { viral pneumonia; } \\
42 \text { Other pneumonia } \\
\text { (CT images) } \\
{ }^{*} \text { The CT-EGFR dataset } \\
\text { was used for auxiliary } \\
\text { training of the } \\
\text { DL system }\end{array}$ & $\begin{array}{l}\text { Lung segmentation; } \\
\text { Using a fully automatic DL model } \\
\text { (DenseNet121-FPN); } \\
\text { suppress the intensities of } \\
\text { non-lung areas inside the } \\
\text { lung ROI; }\end{array}$ & $\begin{array}{l}\text { COVID-19Net } \\
\text { (DenseNet-like } \\
\text { architecture) }\end{array}$ & $\begin{array}{l}\text { Test-set1: } \\
\text { 78.32\% } \\
\text { Test- } \\
\text { set2: 80.12\% }\end{array}$ & $\begin{array}{l}\text { Test-set1: } \\
0.87 \\
\text { Test- } \\
\text { set2: } 0.88\end{array}$ & $\begin{array}{l}\text { Test-set1: } \\
\text { 80.39\% } \\
\text { Test- } \\
\text { set2: } 79.35 \%\end{array}$ & $\begin{array}{l}\text { Test-set1: } \\
\text { 76.61\% } \\
\text { Test- } \\
\text { set2: } 81.16 \%\end{array}$ & (51) \\
\hline $\begin{array}{l}\text { Warman et al. } \\
\text { (2020) }\end{array}$ & "Public sources" & $\begin{array}{l}606 \text { COVID-19 } \\
\text { patients; } \\
224 \text { viral pneumonias } \\
\text { patients; } \\
74 \text { Normal patients } \\
\text { (CT images) }\end{array}$ & Data augmentation & YOLOv3 model & $96.80 \%$ & 0.966 & $98.33 \%$ & $94.95 \%$ & (52) \\
\hline Wu et al. (2020) & Private & $\begin{array}{l}368 \text { COVID-19 } \\
\text { patients; } \\
127 \text { other pneumonia } \\
\text { (CT images) }\end{array}$ & $\begin{array}{l}\text { Lung region in each axial, } \\
\text { coronal and sagittal CT slices } \\
\text { were segmented using threshold } \\
\text { segmentation and morphological } \\
\text { optimization algorithms; } \\
\text { The slice with the most pixels in } \\
\text { the segmented lung area from } \\
\text { each of the axial, coronal and } \\
\text { sagittal views was selected as } \\
\text { the inputs of the deep } \\
\text { learning network; }\end{array}$ & $\begin{array}{l}\text { Multi-view fusion } \\
\text { ResNet50 } \\
\text { architecture }\end{array}$ & $76 \%$ & 0.819 & $81.1 \%$ & $61.5 \%$ & (53) \\
\hline Xu et al. (2020) & $\begin{array}{l}\text { Private "Hospitals in } \\
\text { Zhejiang Province, } \\
\text { China." }\end{array}$ & $\begin{array}{l}219 \text { images from } 110 \\
\text { COVID-19 patients; } \\
224 \text { Influenza-A viral } \\
\text { pneumonia patients; } \\
175 \text { Normal patients } \\
\text { (CT images) }\end{array}$ & $\begin{array}{l}\text { Image processing method base } \\
\text { on } \mathrm{HU} \text { values }\end{array}$ & $\begin{array}{l}\text { 3D CNN } \\
\text { segmentation } \\
\text { model }\end{array}$ & $86.7 \%$ & - & $86.7 \%$ & - & (54) \\
\hline
\end{tabular}


TABLE 1 | Continued

\begin{tabular}{|c|c|c|c|c|c|c|c|c|c|}
\hline \multirow[t]{2}{*}{ Author, year } & \multirow[t]{2}{*}{ Data source } & \multirow{2}{*}{$\begin{array}{l}\text { Data structure and } \\
\text { size }\end{array}$} & \multirow[t]{2}{*}{ Data preprocessing } & \multirow{2}{*}{$\begin{array}{l}\text { Best model } \\
\text { structure(s) }\end{array}$} & \multicolumn{4}{|c|}{ Performance measurements (on the best model) } & \multirow[t]{2}{*}{ References } \\
\hline & & & & & Accuracy & AUC score & Sensitivity & Specificity & \\
\hline Xu et al. (2020) & Private & $\begin{array}{l}432 \text { COVID-19 } \\
\text { patients; } \\
76 \text { other viral } \\
\text { pneumonia; } \\
350 \text { bacterial } \\
\text { pneumonia; } 418 \\
\text { normal patients } \\
\text { (CT images) }\end{array}$ & $\begin{array}{l}\text { Sampling } 5 \text { subsets of CT slices } \\
\text { from all sequential images of one } \\
\text { CT case to picture the infected } \\
\text { lung regions. }\end{array}$ & 3D-Densenet & - & 0.98 & $\begin{array}{l}97.5 \% \\
\text { (differentiating } \\
\text { COVID-19 } \\
\text { from three } \\
\text { types of non- } \\
\text { COVID-19 } \\
\text { cases) } \\
\text { (compared to } \\
79 \% \text { in } \\
\text { human) }\end{array}$ & $\begin{array}{l}89.4 \% \\
\text { (differentiating } \\
\text { COVID-19 } \\
\text { from three } \\
\text { types of non- } \\
\text { COVID-19 } \\
\text { cases) } \\
\text { (compared to } \\
\text { 90\% in } \\
\text { human) }\end{array}$ & (55) \\
\hline Yan et al. (2020) & Private & $\begin{array}{l}416 \text { images from } 206 \\
\text { COVID-19 patients; } \\
412 \text { common } \\
\text { pneumonia patients } \\
\text { (CT images) }\end{array}$ & $\begin{array}{l}\text { Transferring image slices to JPG; } \\
\text { Normalization }\end{array}$ & MSCNN & $97.7 \%$ & 0.962 & $99.5 \%$ & $95.6 \%$ & (56) \\
\hline Yang et al. (2020) & Private & $\begin{array}{l}146 \text { COVID-19 } \\
\text { patients; } \\
149 \text { normal patients } \\
\text { (CT images) }\end{array}$ & $\begin{array}{l}\text { For patients, images containing } \\
\text { round-glasses opacity (GGO), } \\
\text { GGO with consolidation was } \\
\text { selected; for healthy control, } \\
\text { every } 3 \text { slices containing } \\
\text { pulmonary parenchyma were } \\
\text { selected; } \\
\text { Lung windowing is performed } \\
\text { over all image slices; }\end{array}$ & DenseNet & $\begin{array}{l}92 \% \\
\text { (compared to } \\
95 \% \text { in } \\
\text { human) }\end{array}$ & 0.98 & $\begin{array}{l}97 \% \\
\text { (compared to } \\
94 \% \text { in } \\
\text { human) }\end{array}$ & $\begin{array}{l}87 \% \\
\text { (compared to } \\
96 \% \text { in } \\
\text { human) }\end{array}$ & (57) \\
\hline Yu et al. (2020) & Private & $\begin{array}{l}202 \text { COVID-19 patients } \\
\text { (CT images) }\end{array}$ & - & $\begin{array}{l}\text { DenseNet-201 } \\
\text { with the cubic } \\
\text { SVM model }\end{array}$ & $95.2 \%$ & 0.99 & $91.87 \%$ & $96.87 \%$ & (58) \\
\hline $\begin{array}{l}\text { Al-Karawi et al. } \\
(2020)\end{array}$ & "COVID-CT-Dataset" & $\begin{array}{l}275 \text { COVID-19 } \\
\text { patients; } \\
195 \text { normal patients } \\
\text { (CT images) }\end{array}$ & $\begin{array}{l}\text { Adaptive winner filter followed by } \\
\text { inversion; } \\
\text { Feature extraction by } \\
\text { the FFT-spectrum }\end{array}$ & SVM & $95.37 \%$ & - & $95.99 \%$ & $94.76 \%$ & (59) \\
\hline Alom et al. (2020) & $\begin{array}{l}\text { Publicly available } \\
\text { datasets; } \\
\text { "Kaggle repository" }\end{array}$ & $\begin{array}{l}\text { 3,875 pneumonia } \\
\text { patients; } \\
\text { 1,341 normal patients } \\
\text { (X-Ray images) } \\
\text { 178 COVID-19 } \\
\text { patients; } \\
247 \text { normal patients } \\
\text { (CT images) }\end{array}$ & $\begin{array}{l}\text { Data augmentation; } \\
\text { Adaptive Thresholding Approach }\end{array}$ & $\begin{array}{l}\text { IRRCNN model; } \\
\text { NABLA-3 } \\
\text { network model }\end{array}$ & $\begin{array}{l}\text { X-ray images: } \\
84.67 \% \\
\text { CT } \\
\text { images: } 98.78 \%\end{array}$ & 0.93 & - & - & (60) \\
\hline $\begin{array}{l}\text { Barstugan et al. } \\
\text { (2020) }\end{array}$ & $\begin{array}{l}\text { From the Italian Society } \\
\text { of Medical and } \\
\text { Interventional } \\
\text { Radiology }\end{array}$ & $\begin{array}{l}150 \text { COVID-19 patients } \\
\text { (CT images) }\end{array}$ & $\begin{array}{l}13 \text { features were extracted by } \\
\text { Gray Level Size Zone Matrix } \\
\text { (GLSZM) }\end{array}$ & SVM & $98.77 \%$ & - & $97.72 \%$ & $99.67 \%$ & (61) \\
\hline
\end{tabular}


TABLE 1 | Continued

\begin{tabular}{|c|c|c|c|c|c|c|c|c|c|}
\hline \multirow[t]{2}{*}{ Author, year } & \multirow[t]{2}{*}{ Data source } & \multirow{2}{*}{$\begin{array}{l}\text { Data structure and } \\
\text { size }\end{array}$} & \multirow[t]{2}{*}{ Data preprocessing } & \multirow{2}{*}{$\begin{array}{l}\text { Best model } \\
\text { structure(s) }\end{array}$} & \multicolumn{4}{|c|}{ Performance measurements (on the best model) } & \multirow[t]{2}{*}{ References } \\
\hline & & & & & Accuracy & AUC score & Sensitivity & Specificity & \\
\hline Chen et al. (2020) & Private dataset & $\begin{array}{l}25,989 \text { images from } 51 \\
\text { COVID-19 patients; } \\
20,107 \text { images from } 55 \\
\text { normal patients } \\
\text { (retrospective dataset); } \\
\text { 13,911 images from } 27 \\
\text { consecutive patients } \\
\text { (prospective dataset) } \\
\text { (CT images) }\end{array}$ & Filtering & $\begin{array}{l}\text { Deep learning } \\
\text { model }\end{array}$ & $\begin{array}{l}\text { Retrospective } \\
\text { dataset: } \\
\text { 95.24\%; } \\
\text { Prospective } \\
\text { dataset: } \\
\text { 92.59\% } \\
\text { (per patient) }\end{array}$ & - & $\begin{array}{l}\text { Retrospective } \\
\text { dataset: } \\
\text { 100\%; } \\
\text { Prospective } \\
\text { dataset: } \\
100 \% \\
\text { (per patient) }\end{array}$ & $\begin{array}{l}\text { Retrospective } \\
\text { dataset:93.55\%; } \\
\text { Prospective } \\
\text { dataset: } \\
81.82 \% \\
\text { (per patient) }\end{array}$ & (62) \\
\hline Farid et al. (2020) & Kaggle database & $\begin{array}{l}51 \text { COVID-19 patients } \\
\text { (CT images) }\end{array}$ & $\begin{array}{l}\text { Feature extraction (MPEG7 } \\
\text { Histogram Filter, Gabor Image } \\
\text { Filter, Pyramid of } \\
\text { Rotation-Invariant Local Binary } \\
\text { Pattern, Fuzzy 64-bin Histogram } \\
\text { Image Filter); } \\
\text { Feature selection by composite } \\
\text { hybrid feature selection }\end{array}$ & $\begin{array}{l}\text { CHFS-Stacked } \\
\text { (irip, RF) with Naiive } \\
\text { Bayes classifier }\end{array}$ & $96.07 \%$ & - & - & - & (63) \\
\hline Gozes et al. (2020) & $\begin{array}{l}\text { Dataset1:ChainZ; } \\
\text { Dataset2: Private; } \\
\text { Dataset3: ChainZ; }\end{array}$ & $\begin{array}{l}50 \text { suspicious } \\
\text { COVID-19 patients } \\
\text { from dataset1 used for } \\
\text { training; } \\
56 \text { COVID-19 patients; } \\
51 \text { normal patients (CT } \\
\text { images) used } \\
\text { for testing }\end{array}$ & $\begin{array}{l}\text { Data augmentation (rotation, } \\
\text { horizontal flips and cropping) }\end{array}$ & Resnet-50-2D & - & 0.996 & $98.2 \%$ & $92.2 \%$ & (64) \\
\hline Jin et al. (2020) & $\begin{array}{l}\text { Three centers in China; } \\
\text { "LIDC-IDRI;" } \\
\text { "Tianchi-Alibaba;" } \\
\text { "CC-CCII" }\end{array}$ & $\begin{array}{l}\text { 2,529 images from } \\
\text { 1,502 COVID-19 } \\
\text { patients; } \\
\text { 1,338 images from } \\
\text { 1,334 CAP patients; } \\
135 \text { images from } 83 \\
\text { influenza-A/B patients; } \\
258 \text { images from } 258 \\
\text { normal patients } \\
\text { (CT images) }\end{array}$ & - & CNN & - & 0.977 & $90.19 \%$ & $95.76 \%$ & (65) \\
\hline Jin et al. (2020) & $\begin{array}{l}\text { Data from three } \\
\text { different centers in } \\
\text { Wuhan; } \\
\text { Data from three publicly } \\
\text { available databases, } \\
\text { LIDC-IDRI26, } \\
\text { Tianchi-Alibaba27, } \\
\text { and CC-CCI118; }\end{array}$ & $\begin{array}{l}\text { 1,502 COVID-19 } \\
\text { patients; } \\
83 \text { influenza-A/B } \\
\text { patients; } 1,334 \text { CAP } \\
\text { patients except for } \\
\text { influenza; } \\
258 \text { healthy subjects } \\
\text { (CT images) }\end{array}$ & Segmenting lung area with U-net & ResNet152 & - & 0.971 & $90.19 \%$ & $95.76 \%$ & (66) \\
\hline
\end{tabular}


TABLE 1 | Continued

\begin{tabular}{|c|c|c|c|c|c|c|c|c|c|}
\hline \multirow[t]{2}{*}{ Author, year } & \multirow[t]{2}{*}{ Data source } & \multirow{2}{*}{$\begin{array}{l}\text { Data structure and } \\
\text { size }\end{array}$} & \multirow[t]{2}{*}{ Data preprocessing } & \multirow{2}{*}{$\begin{array}{l}\text { Best model } \\
\text { structure(s) }\end{array}$} & \multicolumn{4}{|c|}{ Performance measurements (on the best model) } & \multirow[t]{2}{*}{ References } \\
\hline & & & & & Accuracy & AUC score & Sensitivity & Specificity & \\
\hline $\begin{array}{l}\text { Hosseinzadeh } \\
\text { Kassani et al. } \\
\text { (2020) }\end{array}$ & $\begin{array}{l}\text { COVID-19 X-ray image } \\
\text { database developed by } \\
\text { Cohen JP; } \\
\text { "Kaggle chest X-ray } \\
\text { database;" } \\
\text { "Kaggle RSNA } \\
\text { Pneumonia } \\
\text { Detection dataset" }\end{array}$ & $\begin{array}{l}117 \text { COVID-19 } \\
\text { patients; } \\
117 \text { normal patients } \\
\text { (X-Ray images); } \\
20 \text { COVID-19 patients; } \\
20 \text { normal patients } \\
\text { (CT images) }\end{array}$ & Normalization & $\begin{array}{l}\text { DenseNet121 with } \\
\text { Bagging tree } \\
\text { classifier }\end{array}$ & $99 \%$ & - & $96 \%$ & - & (67) \\
\hline $\begin{array}{l}\text { Ozkaya et al. } \\
\text { (2020) }\end{array}$ & $\begin{array}{l}\text { From the Italian Society } \\
\text { of Medical and } \\
\text { Interventional } \\
\text { Radiology }\end{array}$ & $\begin{array}{l}53 \text { COVID-19 patients } \\
\text { (CT images) }\end{array}$ & $\begin{array}{l}\text { Feature vectors obtained from } \\
\text { Pre-trained VGG-16, GoogleNet } \\
\text { and ResNet-50 networks and } \\
\text { fusion method; } \\
\text { Feature ranking by } t \text {-test method }\end{array}$ & SVM & $98.27 \%$ & - & $98.93 \%$ & $97.60 \%$ & (68) \\
\hline Shi et al. (2020) & $\begin{array}{l}\text { From Tongji Hospital, } \\
\text { Shanghai Public Health } \\
\text { Clinical Center, and } \\
\text { China-Japan Union } \\
\text { Hospital (all in China) }\end{array}$ & $\begin{array}{l}183 \text { COVID-19 } \\
\text { patients; } 5,521 \\
\text { Pneumonia patients } \\
\text { (CT images) }\end{array}$ & $\begin{array}{l}\text { Segmentation by a deep learning } \\
\text { network (VB-Net) }\end{array}$ & $\begin{array}{l}\text { Infection } \\
\text { size-aware } \\
\text { random forest }\end{array}$ & $87.9 \%$ & 0.942 & $90.7 \%$ & $83.3 \%$ & (69) \\
\hline Song et al. (2020) & $\begin{array}{l}\text { From the Renmin } \\
\text { Hospital of Wuhan } \\
\text { University }\end{array}$ & $\begin{array}{l}88 \text { COVID- } 19 \text { patients } \\
\text { (CT images) }\end{array}$ & $\begin{array}{l}\text { We extracted the main regions of } \\
\text { lungs and filled the blank of lung } \\
\text { segmentation with the lung itself }\end{array}$ & $\begin{array}{l}\text { Details Relation } \\
\text { Extraction neural } \\
\text { network }\end{array}$ & $86 \%$ & 0.96 & $96 \%$ & - & (3) \\
\hline Wang et al. (2020) & Private dataset & $\begin{array}{l}44 \text { COVID-19 patients; } \\
55 \text { Pneumonia patients } \\
\text { (CT images) }\end{array}$ & $\begin{array}{l}\text { Random selection of ROI; } \\
\text { Feature extraction using Transfer } \\
\text { Learning }\end{array}$ & $\begin{array}{l}\text { Fully connected } \\
\text { network and } \\
\text { combination of } \\
\text { Decision tree and } \\
\text { Adaboost }\end{array}$ & $82.9 \%$ & 0.90 & $81 \%$ & $84 \%$ & (6) \\
\hline Zheng et al. (2020) & Private dataset & $\begin{array}{l}313 \text { COVID-19 } \\
\text { patients; } 229 \\
\text { non-COVID-19 patients } \\
\text { (CT images) }\end{array}$ & $\begin{array}{l}\text { Data augmentation; Producing } \\
\text { lung masks by a trained UNet }\end{array}$ & $\begin{array}{l}\text { 3D deep } \\
\text { convolutional } \\
\text { neural network }\end{array}$ & $90.8 \%$ & 0.959 & - & - & (70) \\
\hline
\end{tabular}

Data Source: The source(s) that images were acquired from, Data Structure and Size: Number of images, image modalities, sample groups, Data Preprocessing: cleaning, Instance selection, normalization, transformation, feature extraction, selection, etc. The product of data preprocessing is the final training set, Best Model Structure(s): Best machine algorithm or deep learning model reported in the selected paper based on its performance, Performance Measurements (on the best model): The measurement of the model's output performance based on accuracy, sensitivity, specificity, and AUC score. 
TABLE 2 | Characteristics of papers that used X-ray images.

\begin{tabular}{|c|c|c|c|c|c|c|c|c|c|}
\hline \multirow[t]{2}{*}{ Author, year } & \multirow[t]{2}{*}{ Data source } & \multirow[t]{2}{*}{ Data structure and size } & \multirow[t]{2}{*}{ Data preprocessing } & \multirow{2}{*}{$\begin{array}{l}\text { Best model } \\
\text { structure(s) }\end{array}$} & \multicolumn{4}{|c|}{ Performance measurements (on the best model) } & \multirow[t]{2}{*}{ References } \\
\hline & & & & & Accuracy & AUC score & Sensitivity & Specificity & \\
\hline $\begin{array}{l}\text { Alazab et al. } \\
(2020)\end{array}$ & Kaggle database & $\begin{array}{l}70 \text { COVID-19 patients } \\
28 \text { normal patients (X-ray images) }\end{array}$ & Augmented to 1,000 images & VGG-16 & \multicolumn{4}{|c|}{ F1 Score: 0.99} & (16) \\
\hline $\begin{array}{l}\text { Albahli et al. } \\
\text { (2020) }\end{array}$ & $\begin{array}{l}\text { "ChestX-ray8" combined } \\
\text { with the few samples of rare } \\
\text { classes from the Kaggle } \\
\text { challenge }\end{array}$ & $\begin{array}{l}108,948 \text { X-ray images of } 32,717 \text { unique } \\
\text { patients. Including } 15 \text { kinds of chest } \\
\text { disease }\end{array}$ & $\begin{array}{l}\text { Data augmentation (rotation, } \\
\text { height shift, zoom, horizontal flip) }\end{array}$ & ResNet & $89 \%$ & - & - & - & (17) \\
\hline $\begin{array}{l}\text { Albahli et al. } \\
(2020)\end{array}$ & $\begin{array}{l}\text { Open source COVIDx } \\
\text { dataset }\end{array}$ & $\begin{array}{l}850 \text { COVID-19 patients; } \\
500 \text { non-COVID-19 pneumonia cases; } \\
915 \text { normal patients (X-ray images) }\end{array}$ & Data augmentation & InceptionNetV3 & $99.02 \%$ & - & - & - & (18) \\
\hline $\begin{array}{l}\text { Altan et al. } \\
\text { (2020) }\end{array}$ & Not mentioned & $\begin{array}{l}7,980 \text { chest } X \text {-ray image ( } 2,905 \text { real raw } \\
5,075 \text { synthetic chests } X \text {-ray images) }\end{array}$ & $\begin{array}{l}\text { Data augmentation; } \\
\text { The feature matrix is formed by } \\
\text { 2D Curvelet transformation } \\
\text { Coefficients; } \\
\text { Optimizing the coefficients in the } \\
\text { feature matrix with the CSSA }\end{array}$ & Hybrid model & $99.69 \%$ & - & $99.44 \%$ & $99.81 \%$ & (20) \\
\hline $\begin{array}{l}\text { Apostolopoulos } \\
\text { et al. (2020) }\end{array}$ & $\begin{array}{l}\text { COVID-19 X-ray image } \\
\text { database developed by } \\
\text { Cohen JP; } \\
\text { Common Bacterial and Viral } \\
\text { Pneumonia X-ray Images by } \\
\text { Kermany et al.; } \\
\text { Public datasets } \\
\text { (Radiological Society of } \\
\text { North America, } \\
\text { Radiopaedia, and the Italian } \\
\text { Society of Medical and } \\
\text { Interventional Radiology); } \\
\text { "NIH Chest X-ray Dataset" }\end{array}$ & $\begin{array}{l}455 \text { COVID-19 patients; } \\
910 \text { viral pneumonia; } \\
2,540 \text { other pulmonary diseases } \\
\text { (X-ray images) }\end{array}$ & $\begin{array}{l}\text { Data augmentation (randomly } \\
\text { rotated by a maximum of } 10^{\circ} \\
\text { and randomly shifted horizontally } \\
\text { or vertically by a maximum of } 20 \\
\text { pixels toward any direction) }\end{array}$ & MobileNet v2 & $99.18 \%$ & - & $97.36 \%$ & $99.42 \%$ & (21) \\
\hline $\begin{array}{l}\text { Apostolopoulos } \\
\text { et al. (2020) }\end{array}$ & $\begin{array}{l}\text { X-ray images on public } \\
\text { medical Github repositories; } \\
\text { "Radiological Society of } \\
\text { North America;" } \\
\text { "Radiopaedia, and Italian } \\
\text { Society of Medicine and } \\
\text { Interventional Radiology" }\end{array}$ & $\begin{array}{l}\text { Dataset 1: } \\
224 \text { COVID-19 patients; } \\
700 \text { bacterial pneumonia patients; } \\
504 \text { normal patients (X-ray images) } \\
\text { Dataset 2: } \\
224 \text { Covid-19 patients; } \\
714 \text { bacterial and viral pneumonia } \\
\text { patients; } \\
504 \text { normal patients (X-ray images) }\end{array}$ & - & MobileNet v2 & $96.78 \%$ & - & $98.66 \%$ & $96.46 \%$ & (71) \\
\hline $\begin{array}{l}\text { Brunese et al. } \\
\text { (2020) }\end{array}$ & $\begin{array}{l}\text { COVID-19 image data } \\
\text { collection; } \\
\text { COVID-19 X-ray image } \\
\text { database developed by } \\
\text { Cohen JP; } \\
\text { "ChestX-ray8;" } \\
\text { "NIH Chest X-ray Dataset" }\end{array}$ & $\begin{array}{l}250 \text { COVID-19 patients; } \\
\text { 2,753 other pulmonary diseases; } \\
\text { 3,520 normal patients (X-Ray images) }\end{array}$ & $\begin{array}{l}\text { Data augmentation (15 degrees } \\
\text { rotation clockwise or } \\
\text { counterclockwise) }\end{array}$ & VGG-16 & $\begin{array}{l}96 \% \\
\text { (comparison } \\
\text { between } \\
\text { COVID-19 } \\
\text { and other } \\
\text { pulmonary } \\
\text { diseases) }\end{array}$ & - & $\begin{array}{l}87 \% \\
96 \%\end{array}$ & $\begin{array}{l}94 \% \\
98 \%\end{array}$ & (72) \\
\hline
\end{tabular}




\begin{tabular}{|c|c|c|c|c|c|c|c|c|c|}
\hline \multirow[t]{2}{*}{ Author, year } & \multirow[t]{2}{*}{ Data source } & \multirow[t]{2}{*}{ Data structure and size } & \multirow[t]{2}{*}{ Data preprocessing } & \multirow{2}{*}{$\begin{array}{l}\text { Best model } \\
\text { structure(s) }\end{array}$} & \multicolumn{4}{|c|}{ Performance measurements (on the best model) } & \multirow[t]{2}{*}{ References } \\
\hline & & & & & Accuracy & AUC score & Sensitivity & Specificity & \\
\hline $\begin{array}{l}\text { Chowdhury } \\
\text { et al. (2020) }\end{array}$ & $\begin{array}{l}\text { Kaggle chest X-ray } \\
\text { database; } \\
\text { "Italian Society of Medical } \\
\text { and Interventional Radiology } \\
\text { COVID-19 database;" } \\
\text { "Novel Corona Virus } 2019 \\
\text { Dataset;" } \\
\text { GitHub database; } \\
\text { "COVID-19 Chest imaging } \\
\text { at thread reader;" } \\
\text { "RSNA-Pneumonia- } \\
\text { Detection-Challenge" }\end{array}$ & $\begin{array}{l}\text { 423 COVID-19 patients; } \\
\text { 1,485 viral pneumonia patients; } \\
\text { 1,579 normal patients (X-ray images) }\end{array}$ & Data augmentation & CNN & $99.7 \%$ & - & $99.7 \%$ & $99.55 \%$ & (73) \\
\hline $\begin{array}{l}\text { Civit-Masot } \\
\text { et al. (2020) }\end{array}$ & $\begin{array}{l}\text { COVID-19 and Pneumonia } \\
\text { Scans Dataset }\end{array}$ & $\begin{array}{l}132 \text { COVID-19 patients; } \\
132 \text { normal patients; } \\
132 \text { Pneumonia patients (X-ray images) }\end{array}$ & Histogram equalization & VGG16 & $85 \%$ & - & $85 \%$ & $92 \%$ & (74) \\
\hline $\begin{array}{l}\text { Das et al. } \\
\text { (2020) }\end{array}$ & $\begin{array}{l}\text { COVID-19 collection; } \\
\text { "Kaggle CXR collection;" } \\
\text { "Tuberculosis collections;" } \\
\text { "U.S. National Library of } \\
\text { Medicine;" } \\
\text { "National Institutes of } \\
\text { Health;" } \\
\text { Pneumonia collections }\end{array}$ & $\begin{array}{l}162 \text { COVID-19 patients; } \\
1,583 \text { normal patients }\end{array}$ & Histogram matching & $\begin{array}{l}\text { Truncated } \\
\text { Inception Net }\end{array}$ & $\begin{array}{l}100 \% \\
\text { (Pneumonia } \\
\text { collections) }\end{array}$ & 1.0 & $100 \%$ & $100 \%$ & (23) \\
\hline $\begin{array}{l}\text { Elaziz et al. } \\
(2020)\end{array}$ & $\begin{array}{l}\text { COVID-19 X-ray image } \\
\text { database developed by } \\
\text { Cohen JP; } \\
\text { "Chest X-Ray Images } \\
\text { Pneumonia;" Italian Society } \\
\text { of Medical and } \\
\text { Interventional Radiology } \\
\text { COVID-19 DATABASE; }\end{array}$ & $\begin{array}{l}219 \text { COVID-19 patients; } \\
\text { 1,341 negative COVID-19 patients } \\
\text { (X-ray images) }\end{array}$ & $\begin{array}{l}\text { Feature extraction by Fractional } \\
\text { Multichannel Exponent Moments } \\
\text { (FrMEMs); } \\
\text { Feature selection by modified } \\
\text { Manta-Ray } \\
\text { Foraging Optimization based on } \\
\text { differential evolution }\end{array}$ & KNN & 98.09 & - & 98.91 & - & (75) \\
\hline $\begin{array}{l}\text { Hassantabar } \\
\text { et al. (2020) }\end{array}$ & "COVID-CT-Dataset" & $\begin{array}{l}315 \text { COVID-19 patients; } 367 \\
\text { non-COVID-19 patients (X-ray images) }\end{array}$ & - & $\mathrm{CNN}$ & $93.2 \%$ & - & $96.1 \%$ & $99.71 \%$ & (76) \\
\hline $\begin{array}{l}\text { Islam et al. } \\
\text { (2020) }\end{array}$ & $\begin{array}{l}\text { "GitHub;" } \\
\text { "Radiopaedia;" } \\
\text { "Cancer Imaging Archive;" } \\
\text { "Italian Society of } \\
\text { Radiology;" } \\
\text { "Kaggle repository;" } \\
\text { NIH dataset }\end{array}$ & $\begin{array}{l}1,525 \text { COVID-19 patients; } \\
1,525 \text { pneumonia patients; } \\
\text { 1,525 normal patients ( } X \text {-ray images) }\end{array}$ & Normalization & CNN-LSTM & $99.4 \%$ & 0.999 & $99.3 \%$ & $99.2 \%$ & (77) \\
\hline $\begin{array}{l}\text { Khan et al. } \\
\text { (2020) }\end{array}$ & $\begin{array}{l}\text { "Covid-chestxray-dataset" } \\
\text { "Chest X-Ray } \\
\text { Images (Pneumonia)" }\end{array}$ & $\begin{array}{l}284 \text { COVID-19 patients; } \\
330 \text { Pneumonia Bacterial } \\
327 \text { Pneumonia Viral; } \\
310 \text { normal patients (X-ray images) }\end{array}$ & $\begin{array}{l}\text { Random under-sampling (to } \\
\text { overcome the unbalanced data } \\
\text { problem) }\end{array}$ & $\begin{array}{l}\text { CoroNet (based } \\
\text { on Xception) }\end{array}$ & $89.6 \%$ & - & $89.92 \%$ & $96.4 \%$ & (78) \\
\hline
\end{tabular}


TABLE 2 | Continued

\begin{tabular}{|c|c|c|c|c|c|c|c|c|c|}
\hline \multirow[t]{2}{*}{ Author, year } & \multirow[t]{2}{*}{ Data source } & \multirow[t]{2}{*}{ Data structure and size } & \multirow[t]{2}{*}{ Data preprocessing } & \multirow{2}{*}{$\begin{array}{l}\text { Best model } \\
\text { structure(s) }\end{array}$} & \multicolumn{4}{|c|}{ Performance measurements (on the best model) } & \multirow[t]{2}{*}{ References } \\
\hline & & & & & Accuracy & AUC score & Sensitivity & Specificity & \\
\hline $\begin{array}{l}\text { Khuzani et al. } \\
(2020)\end{array}$ & "GitHub" & $\begin{array}{l}140 \text { COVID-19 patients; } \\
140 \text { non-COVID-19 pneumonia } \\
\text { patients; } \\
140 \text { normal patients (X-ray images) }\end{array}$ & $\begin{array}{l}\text { PCA method; } \\
\text { Min-Max Normalization; } \\
\text { Adaptive Histogram Equalization }\end{array}$ & $\mathrm{ML}$ & $94 \%$ & 0.91 & $100 \%$ & - & (79) \\
\hline Ko et al. (2020) & $\begin{array}{l}\text { Private; } \\
\text { Italian Society of Medical } \\
\text { and Interventional Radiology } \\
\text { COVID-19 DATABASE; }\end{array}$ & $\begin{array}{l}\text { 1,194 COVID-19 patients; } \\
\text { 1,442 non-pneumonia patients; } \\
\text { 1,357 Pneumonia patients } \\
\text { (X-ray images) }\end{array}$ & $\begin{array}{l}\text { Data augmentation (rotation, } \\
\text { zoom) }\end{array}$ & $\begin{array}{l}\text { FCONet } \\
\text { (ResNet-50) }\end{array}$ & $99.58 \%$ & - & $99.58 \%$ & $100 \%$ & (80) \\
\hline $\begin{array}{l}\text { Loey et al. } \\
(2020)\end{array}$ & $\begin{array}{l}\text { COVID-19 X-ray image } \\
\text { database developed by } \\
\text { Cohen JP }\end{array}$ & $\begin{array}{l}69 \text { COVID-19 patients; } \\
79 \text { pneumonia bacterial patients; } \\
79\end{array}$ & Data augmentation & Googlenet & $\begin{array}{l}80.56 \% \text { (Four } \\
\text { classes) }\end{array}$ & - & $80.56 \%$ & - & (81) \\
\hline $\begin{array}{l}\text { Mahmud et al. } \\
\text { (2020) }\end{array}$ & Private & $\begin{array}{l}\text { 1,583 normal patients; } \\
1,493 \text { non-COVID viral pneumonia; } \\
2,780 \text { bacterial pneumonia; } 305 \\
\text { COVID-19 patients (X-ray images) }\end{array}$ & - & $\begin{array}{l}\text { CovXNet (CNN } \\
\text { based } \\
\text { architecture) }\end{array}$ & $\begin{array}{l}90.2 \% \\
\text { (multi-class) }\end{array}$ & $\begin{array}{l}0.911 \\
\text { (multi-class) }\end{array}$ & $\begin{array}{l}89.9 \% \\
\text { (multi-class) }\end{array}$ & $\begin{array}{l}89.1 \% \\
\text { (multi-class) }\end{array}$ & (82) \\
\hline $\begin{array}{l}\text { Martínez et al. } \\
\text { (2020) }\end{array}$ & $\begin{array}{l}\text { COVID-19 X-ray image } \\
\text { database developed by } \\
\text { Cohen JP }\end{array}$ & $\begin{array}{l}120 \text { COVID-19 patients; } \\
120 \text { normal patients (X-ray images) }\end{array}$ & $\begin{array}{l}\text { Data augmentation; } \\
\text { Normalization }\end{array}$ & $\begin{array}{l}\text { NASNet-type } \\
\text { convolutional }\end{array}$ & $97 \%$ & - & $97 \%$ & $97 \%$ & (83) \\
\hline $\begin{array}{l}\text { Minaee et al. } \\
\text { (2020) }\end{array}$ & $\begin{array}{l}\text { COVID-19 X-ray image } \\
\text { database developed by } \\
\text { Cohen JP; } \\
\text { "ChexPert dataset" }\end{array}$ & $\begin{array}{l}40 \text { COVID-19 patients; } \\
3,000 \text { normal patients (X-ray images) }\end{array}$ & Regularization & SqueezeNet & $97 \%$ & - & $97.5 \%$ & $97.8 \%$ & (84) \\
\hline $\begin{array}{l}\text { Narayan Das } \\
\text { et al. (2020) }\end{array}$ & $\begin{array}{l}\text { COVID-19 X-ray image } \\
\text { database developed by } \\
\text { Cohen JP; } \\
\text { "ChestX-ray8" }\end{array}$ & $\begin{array}{l}125 \text { COVID-19 patients; } \\
500 \text { pneumonia patients; } \\
500 \text { normal patients (X-ray images) }\end{array}$ & - & Xception & $97.4 \%$ & 0.986 & $97.09 \%$ & $97.29 \%$ & (85) \\
\hline $\begin{array}{l}\text { Nour et al. } \\
(2020)\end{array}$ & $\begin{array}{l}\text { "Public COVID-19 radiology } \\
\text { database;" } \\
\text { "Italian Society of Medical } \\
\text { and Interventional } \\
\text { Radiology;" } \\
\text { "COVID-19 Database;" } \\
\text { "Novel Corona Virus } 2019 \\
\text { Dataset;" } \\
\text { "COVID-19 positive chest } \\
\text { X-ray images from } \\
\text { different articles;" }\end{array}$ & $\begin{array}{l}219 \text { COVID-19 patients; } \\
\text { 1,345 Viral Pneumonia patients; } \\
\text { 1,341 Normal patients (X-ray images) }\end{array}$ & Data augmentation & CNN & $97.14 \%$ & 0.995 & $94.61 \%$ & $98.29 \%$ & (86) \\
\hline $\begin{array}{l}\text { Novitasari et al. } \\
\text { (2020) }\end{array}$ & GitHub and Kaggle & $\begin{array}{l}102 \text { COVID-19 patients; } \\
204 \text { Pneumonia and Normal patients } \\
\text { (X-ray images) }\end{array}$ & $\begin{array}{l}\text { Feature extraction by Googlenet, } \\
\text { Resnet18, Resnet50, } \\
\text { Resnet101; } \\
\text { Feature selection by PCA, Relief; }\end{array}$ & SVM & $\begin{array}{l}97.33 \% \text { (multi - } \\
\text { class) }\end{array}$ & - & $96 \%$ & $98 \%$ & (87) \\
\hline
\end{tabular}


TABLE 2 | Continued

\begin{tabular}{|c|c|c|c|c|c|c|c|c|c|}
\hline \multirow[t]{2}{*}{ Author, year } & \multirow[t]{2}{*}{ Data source } & \multirow[t]{2}{*}{ Data structure and size } & \multirow[t]{2}{*}{ Data preprocessing } & \multirow{2}{*}{$\begin{array}{l}\text { Best model } \\
\text { structure(s) }\end{array}$} & \multicolumn{4}{|c|}{ Performance measurements (on the best model) } & \multirow[t]{2}{*}{ References } \\
\hline & & & & & Accuracy & AUC score & Sensitivity & Specificity & \\
\hline Oh et al. (2020) & $\begin{array}{l}\text { "Japanese Society of } \\
\text { Radiological Technology;" } \\
\text { "SCR database;" } \\
\text { "U.S. National Library } \\
\text { of Medicine" }\end{array}$ & $\begin{array}{l}180 \text { COVID-19 patients; } \\
20 \text { Viral Pneumonia patients; } \\
54 \text { pneumonia bacterial patients; } \\
57 \text { Tuberculosis patients; } \\
191 \text { Normal patients (X-ray images) }\end{array}$ & $\begin{array}{l}\text { Data normalization; } \\
\text { Data type casting; } \\
\text { Histogram equalization; } \\
\text { Gamma correction }\end{array}$ & $\begin{array}{l}\text { (FC)- } \\
\text { DenseNet103 }\end{array}$ & $88.9 \%$ & - & $85.9 \%$ & $96.4 \%$ & (88) \\
\hline $\begin{array}{l}\text { Ozturk et al. } \\
(2020)\end{array}$ & $\begin{array}{l}\text { COVID-19 X-ray image } \\
\text { database developed by } \\
\text { Cohen JP; } \\
\text { "ChestX-ray8;" }\end{array}$ & (X-ray images) & & $\begin{array}{l}\text { DarkCovidNet } \\
\text { inspired by the } \\
\text { DarkNet } \\
\text { architecture }\end{array}$ & $87.02 \%$ & - & $85.35 \%$ & $92.18 \%$ & (89) \\
\hline $\begin{array}{l}\text { Pandit et al. } \\
(2020)\end{array}$ & $\begin{array}{l}\text { COVID-19 X-ray image } \\
\text { database developed by } \\
\text { Cohen JP; } \\
\text { Kaggle chest } \\
\text { X-ray database }\end{array}$ & $\begin{array}{l}224 \text { COVID-19 patients; } \\
700 \text { pneumonia bacterial patients; } \\
504 \text { Normal patients (X-ray images) }\end{array}$ & Data augmentation & VGG-16 & $\begin{array}{l}92.53 \% \\
\text { (Three class } \\
\text { output) }\end{array}$ & - & $86.7 \%$ & $95.1 \%$ & (90) \\
\hline $\begin{array}{l}\text { Panwar et al. } \\
(2020)\end{array}$ & $\begin{array}{l}\text { COVID-19 X-ray image } \\
\text { database developed by } \\
\text { Cohen JP; } \\
\text { Radiopedia.org website; } \\
\text { Kaggle chest } \\
\text { X-ray database }\end{array}$ & $\begin{array}{l}142 \text { COVID-19 patients; } \\
142 \text { other ("Normal" "Bacterial } \\
\text { Pneumonia" and "Viral Pneumonia") } \\
\text { (X-ray images) }\end{array}$ & Data augmentation & nCOVnet & $88.10 \%$ & 0.880 & $97.62 \%$ & $78.57 \%$ & (40) \\
\hline $\begin{array}{l}\text { Pereira et al. } \\
(2020)\end{array}$ & $\begin{array}{l}\text { "RYDLS-20;" } \\
\text { Radiopedia Encyclopedia } \\
\text { "Chest X-ray14" }\end{array}$ & $\begin{array}{l}90 \text { COVID-19 patients; } \\
\text { 1,000 Normal patients; } \\
10 \text { MERS patients; } \\
11 \text { SARS patients; } \\
10 \text { Varicella patients; } \\
12 \text { Streptococcus patients; } \\
11 \text { Pneumocystis patients } \\
\text { (X-ray images) }\end{array}$ & $\begin{array}{l}\text { Resampling algorithms; } \\
\text { Fusion techniques; }\end{array}$ & Pre-trained CNN & & F1 sco & $e=89 \%$ & & (91) \\
\hline $\begin{array}{l}\text { Rahaman et al. } \\
\text { (2020) }\end{array}$ & $\begin{array}{l}\text { COVID-19 X-ray image } \\
\text { database developed by } \\
\text { Cohen JP; "Chest X-Ray } \\
\text { Images (pneumonia)" }\end{array}$ & $\begin{array}{l}260 \text { COVID-19 patients; } \\
300 \text { Pneumonia; } \\
300 \text { Normal patients (X-ray images) }\end{array}$ & $\begin{array}{l}\text { Data augmentation (rotate, shift, } \\
\text { shear, zoom, horizontal and } \\
\text { vertical flip) }\end{array}$ & VGG19 & $89.3 \%$ & - & $89 \%$ & - & (92) \\
\hline $\begin{array}{l}\text { Rahimzadeh } \\
\text { et al. (2020) }\end{array}$ & $\begin{array}{l}\text { "Covid chestxray dataset;" } \\
\text { "RSNA pneumonia } \\
\text { detection challenge" }\end{array}$ & $\begin{array}{l}180 \text { COVID-19 patients; } \\
\text { 6,054 Pneumocystis patients; } \\
\text { 8,851 Normal patients (X-ray images) }\end{array}$ & Data augmentation & $\begin{array}{l}\text { Xception } \\
\text { ResNet50V2 } \\
\text { concatenated }\end{array}$ & $91.4 \%$ & - & $80.53 \%$ & $99.56 \%$ & (93) \\
\hline $\begin{array}{l}\text { Rajaraman } \\
\text { et al. (2020) }\end{array}$ & $\begin{array}{l}\text { Pediatric CXR dataset; } \\
\text { RSNA CXR dataset; } \\
\text { CheXpert CXR dataset; } \\
\text { NIH CXR-14 dataset; } \\
\text { Twitter COVID-19 CXR } \\
\text { dataset; } \\
\text { Montreal COVID-19 } \\
\text { CXR dataset; }\end{array}$ & $\begin{array}{l}\text { 4,683 Bacterial Pneumonia; } \\
\text { 3,883 Viral Pneumonia (X-Ray images) }\end{array}$ & $\begin{array}{l}\text { Segmenting lung area with } \\
\text { dilated dropout U-Net; } \\
\text { Image thresholding to remove } \\
\text { very bright pixels; } \\
\text { In-painting missing pixels using } \\
\text { the surrounding pixel values; } \\
\text { Using median-filter to remove } \\
\text { noise and preserve edges; }\end{array}$ & VGG-16 & $94.05 \%$ & 0.96 & $98.77 \%$ & $86.24 \%$ & (45) \\
\hline
\end{tabular}


TABLE 2 | Continued

\begin{tabular}{|c|c|c|c|c|c|c|c|c|c|}
\hline \multirow[t]{2}{*}{ Author, year } & \multirow[t]{2}{*}{ Data source } & \multirow[t]{2}{*}{ Data structure and size } & \multirow[t]{2}{*}{ Data preprocessing } & \multirow{2}{*}{$\begin{array}{l}\text { Best model } \\
\text { structure(s) }\end{array}$} & \multicolumn{4}{|c|}{ Performance measurements (on the best model) } & \multirow[t]{2}{*}{ References } \\
\hline & & & & & Accuracy & AUC score & Sensitivity & Specificity & \\
\hline $\begin{array}{l}\text { Rajaraman } \\
\text { et al. (2020) }\end{array}$ & $\begin{array}{l}\text { "Pediatric CXR dataset;" } \\
\text { "RSNA CXR dataset;" } \\
\text { "Twitter COVID-19 CXR } \\
\text { dataset;" } \\
\text { "Montreal COVID-19 } \\
\text { CXR dataset" }\end{array}$ & $\begin{array}{l}313 \text { COVID-19 patients; } \\
7,595 \text { pneumonia of unknown type } \\
\text { patients; } \\
2,780 \text { bacterial pneumonia; } \\
\text { 7,595 Normal patients (X-ray images) }\end{array}$ & $\begin{array}{l}\text { Median Filtering; } \\
\text { Normalization; } \\
\text { Standardization }\end{array}$ & Inception-V3 & $99.01 \%$ & 0.997 & $98.4 \%$ & - & (45) \\
\hline $\begin{array}{l}\text { Sethy et al. } \\
\text { (2020) }\end{array}$ & $\begin{array}{l}\text { X-ray images on public } \\
\text { medical Github repositories; } \\
\text { Kaggle chest } \\
\text { X-ray database }\end{array}$ & $\begin{array}{l}127 \text { COVID-19 patients; } \\
127 \text { Pneumonia patients; } \\
127 \text { Normal patients (X-ray images) }\end{array}$ & - & $\begin{array}{l}\text { ResNet50 plus } \\
\text { SVM }\end{array}$ & $98.66 \%$ & - & $95.33 \%$ & - & (94) \\
\hline $\begin{array}{l}\text { Shibly et al. } \\
\text { (2020) }\end{array}$ & $\begin{array}{l}\text { COVID-19 X-ray image } \\
\text { database developed by } \\
\text { Cohen JP; } \\
\text { "RSNA pneumonia } \\
\text { detection challenge } \\
\text { dataset;" } \\
\text { Kaggle chest X-ray } \\
\text { database; } \\
\text { "COVIDx" }\end{array}$ & $\begin{array}{l}183 \text { COVID-19 patients; } \\
\text { 5,551 Pneumonia patients; } \\
\text { 8,066 Normal patients (X-ray images) }\end{array}$ & - & Faster R-CNN & $97.36 \%$ & - & $97.65 \%$ & - & (95) \\
\hline $\begin{array}{l}\text { Togaçar et al. } \\
\text { (2020) }\end{array}$ & $\begin{array}{l}\text { COVID-19 X-ray image } \\
\text { database developed by } \\
\text { Cohen JP; } \\
\text { Kaggle COVID-19 dataset } \\
\text { created by a team of } \\
\text { researchers from Qatar } \\
\text { University, medical doctors } \\
\text { from Bangladesh, and } \\
\text { collaborators from Pakistan } \\
\text { and Malaysia. }\end{array}$ & $\begin{array}{l}295 \text { COVID-19 patients; } \\
98 \text { Pneumonia; } \\
65 \text { normal patients (X-ray images) }\end{array}$ & $\begin{array}{l}\text { Restructuring images using the } \\
\text { Fuzzy Color technique and } \\
\text { stacking them with the original } \\
\text { images; } \\
\text { Feature extracting using deep } \\
\text { learning models (MobileNetV2, } \\
\text { SqueezeNet) using the Social } \\
\text { Mimic optimization method; }\end{array}$ & SVM & $100 \%$ & - & $100 \%$ & $100 \%$ & (96) \\
\hline $\begin{array}{l}\text { Toraman et al. } \\
\text { (2020) }\end{array}$ & $\begin{array}{l}\text { COVID-19 X-ray image } \\
\text { database developed by } \\
\text { Cohen JP }\end{array}$ & $\begin{array}{l}231 \text { COVID-19 patients; } \\
\text { 1,050 Pneumonia patients; } \\
\text { 1,050 Normal patients (X-ray images) }\end{array}$ & Data augmentation; & $\begin{array}{l}\text { Convolutional } \\
\text { capsnet }\end{array}$ & $\begin{array}{l}97.24 \% \\
\text { (Binary class) }\end{array}$ & - & $97.42 \%$ & $97.04 \%$ & (97) \\
\hline
\end{tabular}


TABLE 2 | Continued

\begin{tabular}{|c|c|c|c|c|c|c|c|c|c|}
\hline \multirow[t]{2}{*}{ Author, year } & \multirow[t]{2}{*}{ Data source } & \multirow[t]{2}{*}{ Data structure and size } & \multirow[t]{2}{*}{ Data preprocessing } & \multirow{2}{*}{$\begin{array}{l}\text { Best model } \\
\text { structure(s) }\end{array}$} & \multicolumn{4}{|c|}{ Performance measurements (on the best model) } & \multirow[t]{2}{*}{ References } \\
\hline & & & & & Accuracy & AUC score & Sensitivity & Specificity & \\
\hline $\begin{array}{l}\text { Tsiknakis et al. } \\
\text { (2020) }\end{array}$ & $\begin{array}{l}\text { COVID-19 X-ray image } \\
\text { database developed by } \\
\text { Cohen JP; } \\
\text { Dataset originated from the } \\
\text { QUIBIM imagingcovid19 } \\
\text { platform database and } \\
\text { various public repositories, } \\
\text { including RSNA, IEEE, } \\
\text { RadioGyan and the British } \\
\text { Society of Thoracic Imaging; } \\
\text { Publicly available } \\
\text { X-ray dataset of patients } \\
\text { with pneumonia; }\end{array}$ & $\begin{array}{l}137 \text { COVID-19 patients; } \\
150 \text { Virus Pneumonia; } \\
150 \text { Bacteria Pneumonia; } \\
150 \text { normal patients (X-ray images) }\end{array}$ & $\begin{array}{l}\text { Data augmentation (rotation, } \\
\text { shear, zoom) }\end{array}$ & Inception V3 & $\begin{array}{l}76 \% \\
\text { (multi-class) }\end{array}$ & $\begin{array}{l}0.93 \\
\text { (multi-class) }\end{array}$ & $\begin{array}{l}93 \% \\
\text { (multi-class) }\end{array}$ & $\begin{array}{l}91.8 \% \\
\text { (multi-class) }\end{array}$ & (98) \\
\hline $\begin{array}{l}\text { Tuncer et al. } \\
(2020)\end{array}$ & $\begin{array}{l}\text { GitHub website; } \\
\text { Kaggle chest } \\
\text { X-ray database }\end{array}$ & $\begin{array}{l}87 \text { COVID-19 patients; } \\
234 \text { Normal patients (X-ray images) }\end{array}$ & $\begin{array}{l}\text { Converting X-ray image to } \\
\text { grayscale; } \\
\text { ResEXLBP and IRF } \\
\text { based method }\end{array}$ & SVM & $100 \%$ & - & $98.29 \%$ & $100 \%$ & (99) \\
\hline $\begin{array}{l}\text { Ucar et al. } \\
(2020)\end{array}$ & $\begin{array}{l}\text { "COVID chest X-ray } \\
\text { dataset;" "Kaggle chest } \\
\text { X-ray pneumonia dataset;" }\end{array}$ & $\begin{array}{l}403 \text { COVID-19 patients; } \\
721 \text { normal patients (X-ray images) }\end{array}$ & $\begin{array}{l}\text { Data augmentation (noise, shear, } \\
\text { brightness increase, brightness } \\
\text { decrease) }\end{array}$ & $\begin{array}{l}\text { Bayes- } \\
\text { SqueezeNet }\end{array}$ & $\begin{array}{l}98.26 \% \\
\text { (multi-class) }\end{array}$ & - & - & $\begin{array}{l}99.13 \% \\
\text { (multi-class) }\end{array}$ & $(100)$ \\
\hline $\begin{array}{l}\text { Vaid et al. } \\
(2020)\end{array}$ & $\begin{array}{l}\text { Set of lately published } \\
\text { articles; } \\
\mathrm{NIH} \text { dataset }\end{array}$ & $\begin{array}{l}181 \text { COVID-19 patients; } \\
364 \text { Normal patients (X-ray images) }\end{array}$ & Normalization & VGG-19 & $96.3 \%$ & - & $97.1 \%$ & - & (101) \\
\hline $\begin{array}{l}\text { Waheed et al. } \\
\text { (2020) }\end{array}$ & $\begin{array}{l}\text { "IEEE Covid Chest X-ray } \\
\text { dataset;" } \\
\text { "COVID-19 Radiography } \\
\text { Database" } \\
\text { "COVID-19 Chest } \\
\text { X-ray Dataset;" }\end{array}$ & $\begin{array}{l}403 \text { COVID-19 patients; } \\
721 \text { normal patients (X-ray images) }\end{array}$ & $\begin{array}{l}\text { Data augmentation using } \\
\text { CovidGAN }\end{array}$ & VGG16 & $95 \%$ & - & $90 \%$ & $97 \%$ & (102) \\
\hline $\begin{array}{l}\text { Yildirim et al. } \\
(2020)\end{array}$ & $\begin{array}{l}\text { "COVID-19 Chest X-Ray } \\
\text { dataset;" } \\
\text { Kaggle chest } \\
\text { X-ray database }\end{array}$ & $\begin{array}{l}136 \text { COVID-19 patients; } \\
162 \text { Pneumonia patients; } \\
245 \text { Normal patients (X-ray images) }\end{array}$ & - & Hybrid model & $96.30 \%$ & - & $96.30 \%$ & $98.73 \%$ & (103) \\
\hline $\begin{array}{l}\text { Yoo et al. } \\
\text { (2020) }\end{array}$ & $\begin{array}{l}\text { "COVID-Chest } \\
\text { XrayDataset;" } \\
\text { Eastern Asian Hospital; } \\
\text { Shenzen data; }\end{array}$ & $\begin{array}{l}162 \text { COVID-19 Patients; } \\
162 \text { TB patients; } \\
162 \text { Non-TB patients (X-ray images) }\end{array}$ & $\begin{array}{l}\text { Data augmentation (rotated, } \\
\text { translated, and horizontally } \\
\text { flipped) }\end{array}$ & ResNet18 & $\begin{array}{l}\text { 95\% Average } \\
\text { of (COVID- } \\
19 / T B \text { ) and } \\
\text { (COVID- } \\
19 / \text { non-TB) }\end{array}$ & $\begin{array}{l}0.95 \text { Average } \\
\text { of (COVID- } \\
19 / T B) \text { and } \\
\text { (COVID- } \\
19 / \text { non-TB) }\end{array}$ & $\begin{array}{l}\text { 97\% Average } \\
\text { of (COVID- } \\
19 / \text { TB) and } \\
\text { (COVID- } \\
\text { 19/non-TB) }\end{array}$ & $\begin{array}{l}\text { 93\% Average } \\
\text { of (COVID- } \\
19 / \text { TB) and } \\
\text { (COVID- } \\
\text { 19/non-TB) }\end{array}$ & (104) \\
\hline $\begin{array}{l}\text { Ghoshal et al. } \\
(2020)\end{array}$ & $\begin{array}{l}\text { COVID-19 X-ray image } \\
\text { database developed by } \\
\text { Cohen JP; } \\
\text { "Kaggle chest } \\
\text { X-ray database" }\end{array}$ & $\begin{array}{l}68 \text { COVID-19 patients; } \\
\text { 2,786 Bacterial } \\
\text { Pneumonia patients; } \\
\text { 1,504 Viral Pneumonia patients; } \\
\text { 1,583 normal patients (X-Ray images) }\end{array}$ & $\begin{array}{l}\text { Standardization; } \\
\text { Data augmentation }\end{array}$ & $\begin{array}{l}\text { Bayesian } \\
\text { ResNet50V2 } \\
\text { model }\end{array}$ & $89.82 \%$ & - & - & - & (105) \\
\hline
\end{tabular}


TABLE 2 | Continued

\begin{tabular}{|c|c|c|c|c|c|c|c|c|c|}
\hline \multirow[t]{2}{*}{ Author, year } & \multirow[t]{2}{*}{ Data source } & \multirow[t]{2}{*}{ Data structure and size } & \multirow[t]{2}{*}{ Data preprocessing } & \multirow{2}{*}{$\begin{array}{l}\text { Best model } \\
\text { structure(s) }\end{array}$} & \multicolumn{4}{|c|}{ Performance measurements (on the best model) } & \multirow[t]{2}{*}{ References } \\
\hline & & & & & Accuracy & AUC score & Sensitivity & Specificity & \\
\hline $\begin{array}{l}\text { Hall et al. } \\
(2020)\end{array}$ & $\begin{array}{l}\text { "X-ray images on public } \\
\text { medical Github } \\
\text { repositories;" } \\
\text { "Radiopaedia;" } \\
\text { "Italian Society of Medical } \\
\text { and Interventional } \\
\text { Radiology (SIRM)" }\end{array}$ & $\begin{array}{l}135 \text { COVID-19 patients; } \\
320 \text { Viral and Bacterial Pneumonia } \\
\text { patients (X-Ray images) }\end{array}$ & Data augmentation & $\begin{array}{l}\text { Resnet50 and } \\
\text { VGG16 plus } \\
\text { CNN }\end{array}$ & $91.24 \%$ & 0.94 & - & - & (106) \\
\hline $\begin{array}{l}\text { Hammoudi } \\
\text { et al. (2020) }\end{array}$ & $\begin{array}{l}\text { "Chest XRay Images } \\
\text { (Pneumonia) dataset;" } \\
\text { COVID-19 X-ray image } \\
\text { database developed by } \\
\text { Cohen JP; }\end{array}$ & $\begin{array}{l}148 \text { Bacterial pneumonia; } \\
148 \text { Viral pneumonia; } \\
148 \text { Normal patients (X-Ray Images) }\end{array}$ & - & DenseNet169 & $95.72 \%$ & - & - & - & $(107)$ \\
\hline $\begin{array}{l}\text { El-Din Hemdan } \\
\text { et al. (2020) }\end{array}$ & $\begin{array}{l}\text { COVID-19 X-ray image } \\
\text { database developed by } \\
\text { Cohen JP; } \\
\text { COVID-19 X-ray image } \\
\text { database by Dr. } \\
\text { Adrian Rosebrock }\end{array}$ & $\begin{array}{l}25 \text { COVID-19 patients; } \\
25 \text { normal patients (X-Ray images) }\end{array}$ & $\begin{array}{l}\text { Scaling to } 224^{*} 224 \text { pixels; } \\
\text { One-hot encoding }\end{array}$ & $\begin{array}{l}\text { COVIDX-Net } \\
\text { (VGG19 and } \\
\text { DenseNet201 } \\
\text { models) }\end{array}$ & $\begin{array}{l}\text { VGG19= } \\
\text { 90\%; } \\
\text { DenseNet201 } \\
=90 \%\end{array}$ & $\begin{array}{l}\text { VGG19= } \\
0.90 ; \\
\text { DenseNet201 } \\
=0.90\end{array}$ & $\begin{array}{l}\text { VGG19= } \\
\text { 100\%; } \\
\text { DenseNet201 } \\
=100 \%\end{array}$ & - & (108) \\
\hline $\begin{array}{l}\text { Jain et al. } \\
\text { (2020) }\end{array}$ & $\begin{array}{l}\text { "Chest XRay Images } \\
\text { (Pneumonia) dataset;" } \\
\text { COVID-19 X-ray image } \\
\text { database developed by } \\
\text { Cohen JP; }\end{array}$ & $\begin{array}{l}250 \text { COVID-19 patients; } \\
300 \text { Bacterial pneumonia; } \\
350 \text { Viral pneumonia; } \\
315 \text { Normal patients (X-Ray Images) }\end{array}$ & $\begin{array}{l}\text { Normalize images according to } \\
\text { the images in the ImageNet } \\
\text { database; } \\
\text { Data augmentation (rotation and } \\
\text { Gaussian blur); }\end{array}$ & ResNet50 & $97.77 \%$ & - & $97.14 \%$ & - & (109) \\
\hline $\begin{array}{l}\text { Luz et al. } \\
\text { (2020) }\end{array}$ & $\begin{array}{l}\text { "COVIDx dataset;" } \\
\text { "RSNA Pneumonia } \\
\text { Detection Challenge } \\
\text { dataset;" } \\
\text { "COVID-19 image } \\
\text { data collection" }\end{array}$ & $\begin{array}{l}183 \text { COVID-19 patients; } \\
\text { 5,521 Pneumonia patients; } \\
\text { 8,066 normal patients (X-Ray images) }\end{array}$ & $\begin{array}{l}\text { Intensity normalization; } \\
\text { Data augmentation }\end{array}$ & EfficientNet B3 & $93.9 \%$ & - & $96.8 \%$ & - & $(110)$ \\
\hline $\begin{array}{l}\text { Ozkaya et al. } \\
\text { (2020) }\end{array}$ & $\begin{array}{l}\text { From the Italian Society of } \\
\text { Medical and Interventional } \\
\text { Radiology }\end{array}$ & 53 COVID-19 patients (CT images) & $\begin{array}{l}\text { Feature vectors obtained from } \\
\text { Pre-trained VGG-16, GoogleNet } \\
\text { and ResNet-50 networks and } \\
\text { fusion method; } \\
\text { Feature ranking by } t \text {-test method }\end{array}$ & SVM & $98.27 \%$ & - & $98.93 \%$ & $97.60 \%$ & (68) \\
\hline
\end{tabular}


TABLE 2 | Continued

\begin{tabular}{|c|c|c|c|c|c|c|c|c|c|}
\hline \multirow[t]{2}{*}{ Author, year } & \multirow[t]{2}{*}{ Data source } & \multirow[t]{2}{*}{ Data structure and size } & \multirow[t]{2}{*}{ Data preprocessing } & \multirow{2}{*}{$\begin{array}{l}\text { Best model } \\
\text { structure(s) }\end{array}$} & \multicolumn{4}{|c|}{ Performance measurements (on the best model) } & \multirow[t]{2}{*}{ References } \\
\hline & & & & & Accuracy & AUC score & Sensitivity & Specificity & \\
\hline $\begin{array}{l}\text { Ozturk et al. } \\
(2020)\end{array}$ & $\begin{array}{l}\text { "covid-chestxray-dataset } \\
\text { available at: https://github. } \\
\text { com/ieee8023/covid- } \\
\text { chestxray-dataset" }\end{array}$ & $\begin{array}{l}4 \text { ARds images, } 101 \text { COVID images, } 2 \\
\text { No finding images, } 2 \\
\text { pneumocystis-pneumonia images, } 11 \\
\text { Sars images, and } 6 \text { streptococcus } \\
\text { (X-Ray images) }\end{array}$ & $\begin{array}{l}\text { Data augmentation; SMOTE } \\
\text { oversampling; creating feature } \\
\text { vectors with sAE and PCA; } \\
\text { feature extraction by feature } \\
\text { vectors, Gray Level } \\
\text { Co-occurrence Matrix, Local } \\
\text { Binary Gray Level Co-occurrence } \\
\text { Matrix, Gray Level Run Length } \\
\text { Matrix, and Segmentation-based } \\
\text { Fractal Texture Analysis }\end{array}$ & SVM & $94.23 \%$ & 0.99 & $91.88 \%$ & $98.54 \%$ & (111) \\
\hline $\begin{array}{l}\text { Wang et al. } \\
\text { (2020) }\end{array}$ & COVIDx dataset & $\begin{array}{l}266 \text { COVID-19 patients; 5,536 } \\
\text { Pneumonia patients; 8,066 normal } \\
\text { patients (X-Ray images) }\end{array}$ & - & $\begin{array}{l}\text { COVID-Net } \\
\text { Network } \\
\text { Architecture using } \\
\text { a "lightweight } \\
\text { residual } \\
\text { projection- } \\
\text { expansion- } \\
\text { projection- } \\
\text { extension design } \\
\text { pattern" } \\
\text { (Customized } \\
\text { CNN) }\end{array}$ & $93.3 \%$ & & $91.0 \%$ & - & (1) \\
\hline $\begin{array}{l}\text { Zhang et al. } \\
(2020)\end{array}$ & X-COVID, OpenCOVID & $\begin{array}{l}599 \text { COVID-19 patients; } 2,107 \\
\text { non-COVID-19 patients (non-viral } \\
\text { pneumonia and healthy) (X-Ray images) }\end{array}$ & $\begin{array}{l}\text { Data augmentation; Feature } \\
\text { extraction using EfficientNet }\end{array}$ & $\begin{array}{l}\text { Confidence- } \\
\text { aware anomaly } \\
\text { detection }\end{array}$ & $78.57 \%$ & 0.844 & $77.13 \%$ & $78.97 \%$ & $(112)$ \\
\hline
\end{tabular}

Data Source: The source(s) that images were acquired from, Data Structure and Size: Number of images, image modalities, sample groups, Data Preprocessing: cleaning, Instance selection, normalization, transformation, feature extraction, selection, etc. The product of data preprocessing is the final training set, Best Model Structure(s): Best machine algorithm or deep learning model reported in the selected paper based on its performance, Performance Measurements (on the best model): The measurement of the model's output performance based on accuracy, sensitivity, specificity, and AUC score. 


\section{DISCUSSION}

Machine and deep learning methods have been proven as valuable strategies to assess massive high-dimensional characteristics of medical images. CT or X-Ray findings of COVID-19 patients have similarities with other atypical and viral pneumonia diseases. Therefore, machine and deep learning methods might facilitate automatic discrimination of COVID19 from other pneumonia conditions. The differential diagnosis of COVID also includes drug-induced diseases or immune pneumonitis. However, most of the studies reviewed here lack these kinds of samples. This point is the limitation of these studies. Different methods, such as Ensemble, VGG16, ResNet, InceptionNetV3, MobileNet v2, Xception, CNN, VGG16, Truncated Inception Net, and KNN, have been used for the purpose of assessment of chest images of COVID-19 patients. Notably, the application of these methods on X-rays has offered promising results. Such a finding is particularly important since X-rays are easily accessible and low cost. These methods not only can diagnose COVID-19 patients from non-COVID pneumonia cases, but can also predict the severity of COVID-19 pneumonia and the risk of short-term mortality. In spite of the low expense of X-ray compared with CT images, the numbers of studies that assessed these two types of imaging using machine/deep learning methods are not meaningfully different. However, few studies have used these methods on both types of imaging $(25,29,40)$. CNN-based methods have achieved accuracy values above $99 \%$ in classifying COVID-19 patients from other cases of pneumonia or related disorders, as reported by several independent studies, suggesting these strategies as screening methods for initial evaluation of COVID-19 cases.

Although both deep learning and machine learning strategies can be used for the mentioned purpose, they differ in some respects. For instance, deep learning methods usually need a large amount of labeled training data to make a concise conclusion. However, machine learning can apply a small amount of data delivered by users. Moreover, deep learning methods need highperformance hardware. Machine learning, on the other hand, needs features to be precisely branded by users, deep learning generates novel features by itself, thus requires more time to train. Machine learning classifies tasks into small fragments and subsequently combines obtained results into one conclusion, whereas deep learning resolves the problems using end-toend principles.

Several studies have diagnosed COVID-19 patients through the application of machine learning methods rather than using deep learning methods by retrieving the features from the images. These studies have yielded high recognition outcomes and have the advantage of high learning speed (12). Preprocessing is an essential step for reducing the impacts of intensity variations in CT slices and getting rid of noise. Subsequent thresholding and morphological operations have also enhanced the analytical performance. Data augmentation and histogram equalization are among the most applied preprocessing methods.
One of the most promising approaches used in the included studies was transfer learning. Transfer learning is defined as using model knowledge on a huge dataset (which is referred to as the "pre-trained model") and transferring it to use on a new problem. This is very useful in settings like medical imaging, where there is a limited number of labeled data (113). Previous studies showed favorable outcomes of the transfer learning approaches in medical imaging tasks (114, 115). Among the included studies, Bridge et al. (25) even reached $100 \%$ classification accuracy on COVID-19 using the pre-trained InceptionV3.

The availability of public databases of CT and X-ray images of patients with COVID-19 has facilitated the application of machine learning methods on large quantities of clinical images and execution of training and verification steps. However, since these images have come from various institutes using different scanners, preprocessing of the obtained data is necessary to make them uniform and facilitate further analysis (12). Appraisal of demographic and clinical data of COVID-19 patients and their association with CT/ X-ray images features as well as the accuracy of machine learning prediction methods would provide more valuable information in the stratification of COVID-19 patients. Moreover, one of the major challenges of deep learning models in medical applications is its unexplainable features due to its black-box nature, which should be solved (116). Future studies can focus on approaches that provide interpretation besides black-box predictions.

\section{CONCLUSION}

Deep and machine learning methods have high accuracy in the differentiation of COVID-19 from non-COVID-19 pneumonia based on chest images. These techniques have facilitated the automatic evaluation of these images. However, deep learning methods suffer from the absence of transparency and interpretability, as it is not possible to identify the exact imaging feature that has been applied to define the output (13). As no single strategy has the capacity to distinguish all pulmonary disorders based merely on the imaging presentation on chest CT scans, the application of multidisciplinary approaches is suggested for overcoming diagnostic problems (13).

\section{DATA AVAILABILITY STATEMENT}

The original contributions presented in the study are included in the article/supplementary material, further inquiries can be directed to the corresponding authors.

\section{AUTHOR CONTRIBUTIONS}

HM-R, MN, and AG-L collected the data and designed the tables. MT and SG-F designed the study, wrote the draft, and revised it. All the authors read the draft and approved the submitted version. 


\section{REFERENCES}

1. Wang L, Wong A. COVID-Net: a tailored deep convolutional neural network design for detection of covid-19 cases from chest X-ray images. arXiv. (2020) Preprint arXiv:200309871. doi: 10.1038/s41598-020-76550-Z

2. Ghafouri-Fard S, Noroozi R, Vafaee R, Branicki W, Posipiech E, Pyrc $\mathrm{K}$, et al. Effects of host genetic variations on response to, susceptibility and severity of respiratory infections. Biomed Pharmacother. (2020) 128:110296. doi: 10.1016/j.biopha.2020.110296

3. Song Y, Zheng S, Li L, Zhang X, Zhang X, Huang Z, et al. Deep learning enables accurate diagnosis of novel coronavirus (COVID-19) with CT images. medRxiv. (2020).

4. Samsami M, Mehravaran E, Tabarsi P, Javadi A, Arsang-Jang S, Komaki A, et al. Clinical and demographic characteristics of patients with COVID-19 infection: statistics from a single hospital in Iran. Human Antibodies. (2020) 1-6. doi: 10.3233/HAB-200428

5. Ghafouri-Fard S, Noroozi R, Omrani MD, Branicki W, Pośpiech E, Sayad A, et al. Angiotensin converting enzyme: a review on expression profile and its association with human disorders with special focus on SARS-CoV-2 infection. Vascular Pharmacol. (2020) 130:106680. doi: 10.1016/j.vph.2020.106680

6. Wang S, Kang B, Ma J, Zeng X, Xiao M, Guo J, et al. A deep learning algorithm using CT images to screen for Corona Virus Disease (COVID-19). medRxiv. (2020) 14:1-9. doi: 10.1101/2020.02.14.20023028

7. Fang Y, Zhang H, Xie J, Lin M, Ying L, Pang P, et al. Sensitivity of chest CT for COVID-19: comparison to RT-PCR. Radiology. (2020) 296:12. doi: 10.1148/radiol.2020200432

8. Zhang J, Tian S, Lou J, Chen Y. Familial cluster of COVID-19 infection from an asymptomatic. Crit Care. (2020) 24:1-3. doi: 10.1186/s13054-020-2817-7

9. Lei Y, Zhang H-W, Yu J, Patlas MN. COVID-19 Infection: Early Lessons. Los Angeles, CA: Sage (2020).

10. Rousan LA, Elobeid E, Karrar M, Khader Y. Chest X-ray findings and temporal lung changes in patients with COVID-19 pneumonia. BMC Pulmonary Med. (2020) 20:1-9. doi: 10.1186/s12890-020-01286-5

11. Bai HX, Hsieh B, Xiong Z, Halsey K, Choi JW, Tran TML, et al. Performance of radiologists in differentiating COVID-19 from viral pneumonia on chest CT. Radiology. (2020) 296:1-8. doi: 10.1148/radiol.2020200823

12. Ozsahin I, Sekeroglu B, Musa MS, Mustapha MT, Uzun Ozsahin D. Review on diagnosis of COVID-19 from chest CT images using artificial intelligence. Comput Math Methods Med. (2020) 2020:1-10. doi: 10.1155/2020/9756518

13. Li L, Qin $\mathrm{L}, \mathrm{Xu} Z$, Yin $\mathrm{Y}$, Wang $\mathrm{X}$, Kong B, et al. Using artificial intelligence to detect COVID-19 and community-acquired pneumonia based on pulmonary CT: evaluation of the diagnostic accuracy. Radiology. (2020) 296:E65-71. doi: 10.1148/radiol.2020200905

14. rekha Hanumanthu S. Role of intelligent computing in COVID-19 prognosis: a state-of-the-art review. Chaos Solitons Fractals. (2020) 138:109947. doi: 10.1016/j.chaos.2020.109947

15. Abbasian Ardakani A, Acharya UR, Habibollahi S, Mohammadi A. COVIDiag: a clinical CAD system to diagnose COVID19 pneumonia based on CT findings. Eur Radiol. (2020) 31:1-10. doi: 10.1007/s00330-020-07087-y

16. Alazab M, Awajan A, Mesleh A, Abraham A, Jatana V, Alhyari S. COVID19 prediction and detection using deep learning. Int J Comput Information Syst Indus Manage Appl. (2020) 12:168-81. doi: 10.1016/j.chaos.2020. 110338

17. Albahli S. Efficient GAN-based Chest Radiographs (CXR) augmentation to diagnose coronavirus disease pneumonia. Int J Med Sci. (2020) 17:1439-48. doi: 10.7150/ijms.46684

18. Albahli S, Albattah W. Detection of coronavirus disease from X-ray images using deep learning and transfer learning algorithms. J Xray Sci Technol. (2020) 28:841-50. doi: 10.3233/XST-200720

19. Alsharman N, Jawarneh I. GoogleNet CNN neural network towards chest CT-coronavirus medical image classification. J Comput Sci. (2020) 16:620-5 doi: 10.3844/jcssp.2020.620.625

20. Altan A, Karasu S. Recognition of COVID-19 disease from X-ray images by hybrid model consisting of $2 \mathrm{D}$ curvelet transform, chaotic salp swarm algorithm and deep learning technique. Chaos Solitons Fractals. (2020) 140:110071. doi: 10.1016/j.chaos.2020.110071
21. Apostolopoulos ID, Aznaouridis SI, Tzani MA. Extracting possibly representative COVID-19 biomarkers from X-ray images with deep learning approach and image data related to pulmonary diseases. J Med Biol Eng. (2020) 40:1-8. doi: 10.1007/s40846-020-00529-4

22. Ardakani AA, Kanafi AR, Acharya UR, Khadem N, Mohammadi A. Application of deep learning technique to manage COVID19 in routine clinical practice using CT images: results of 10 convolutional neural networks. Comput Biol Med. (2020) 121:103795. doi: 10.1016/j.compbiomed.2020.103795

23. Das D, Santosh KC, Pal U. Truncated inception net: COVID-19 outbreak screening using chest X-rays. Phys Eng Sci Med. (2020) 43:1-11. doi: 10.21203/rs.3.rs-20795/v1

24. Aswathy SU, Jarin T, Mathews R, Nair LM, Rroan M. CAD systems for automatic detection and classification of COVID-19 in nano CT lung image by using machine learning technique. Int J Pharm Res. (2020) 12:1865-70. doi: 10.31838/ijpr/2020.12.02.247

25. Bridge J, Meng Y, Zhao Y, Du Y, Zhao M, Sun R, et al. Introducing the GEV activation function for highly unbalanced data to develop COVID-19 diagnostic models. IEEE J Biomed Health Inform. (2020) 24:110. doi: $10.1109 /$ JBHI.2020.3012383

26. Butt C, Gill J, Chun D, Babu BA. Deep learning system to screen coronavirus disease 2019 pneumonia. Appl Intell. (2020) 6:1-7. doi: 10.1007/s10489-020-01714-3

27. Dey N, Rajinikanth V, Fong SJ, Kaiser MS, Mahmud M. Social group optimization-assisted Kapur's entropy and morphological segmentation for automated detection of COVID-19 infection from computed tomography images. Cognit Comput. (2020) 12:1-13. doi: 10.20944/preprints202005.0052.v1

28. Kermany D, Zhang K, Goldbaum M. Labeled optical coherence tomography (OCT) and Chest X-Ray images for classification. Mendeley Data. (2018) 2. doi: 10.17632/RSCBJBR9SJ.2

29. El Asnaoui K, Chawki Y. Using X-ray images and deep learning for automated detection of coronavirus disease. J Biomol Struct Dyn. (2020) 1-12. doi: 10.1080/07391102.2020.1767212

30. Han Z, Wei B, Hong Y, Li T, Cong J, Zhu X, et al. Accurate screening of COVID-19 using attention-based deep 3D multiple instance learning. IEEE Trans Med Imaging. (2020) 39:2584-94. doi: 10.1109/TMI.2020.2996256

31. Harmon SA, Sanford TH, Xu S, Turkbey EB, Roth H, Xu Z, et al. Artificial intelligence for the detection of COVID-19 pneumonia on chest CT using multinational datasets. Nat Commun. (2020) 11:4080. doi: 10.1038/s41467-020-17971-2

32. Hasan AM, Al-Jawad MM, Jalab HA, Shaiba H, Ibrahim RW, Al-Shamasneh AR. Classification of Covid-19 coronavirus, pneumonia and healthy lungs in CT scans using Q-deformed entropy and deep learning features. Entropy. (2020) 22:517. doi: 10.3390/e22050517

33. Hu S, Gao Y, Niu Z, Jiang Y, Li L, Xiao X, et al. Weakly supervised deep learning for COVID-19 infection detection and classification from CT images. IEEE Access. (2020) 8:118869-83. doi: 10.1109/ACCESS.2020.3005510

34. Jaiswal A, Gianchandani N, Singh D, Kumar V, Kaur M. Classification of the COVID-19 infected patients using DenseNet201 based deep transfer learning. J Biomol Struct Dyn. (2020) 1-8. doi: 10.1080/07391102.2020.1788642

35. Kang H, Xia L, Yan F, Wan Z, Shi F, Yuan H, et al. Diagnosis of coronavirus disease 2019 (COVID-19) with structured latent multiview representation learning. IEEE Trans Med Imaging. (2020) 39:2606-14. doi: 10.1109/TMI.2020.2992546

36. Lessmann N, Sánchez CI, Beenen L, Boulogne LH, Brink M, Calli E, et al. Automated assessment of CO-RADS and chest CT severity scores in patients with suspected COVID-19 using artificial intelligence. Radiology. (2020) 202439.

37. Li Y, Dong W, Chen J, Cao S, Zhou H, Zhu Y, et al. Efficient and effective training of COVID-19 classification networks with self-supervised dual-track learning to rank. IEEE J Biomed Health Inform. (2020) 24:110. doi: 10.1109/JBHI.2020.3018181

38. Liu C, Wang X, Liu C, Sun Q, Peng W. Differentiating novel coronavirus pneumonia from general pneumonia based 
on machine learning. Biomed Eng Online. (2020) 19:66. doi: 10.1186/s12938-020-00809-9

39. Mei X, Lee HC, Diao KY, Huang M, Lin B, Liu C, et al. Artificial intelligenceenabled rapid diagnosis of patients with COVID-19. Nat Med. (2020) 26:1224-8. doi: 10.1038/s41591-020-0931-3

40. Panwar H, Gupta PK, Siddiqui MK, Morales-Menendez R, Singh V. Application of deep learning for fast detection of COVID19 in X-Rays using nCOVnet. Chaos Solitons Fractals. (2020) 138:109944. doi: 10.1016/j.chaos.2020.109944

41. Pathak Y, Shukla PK, Tiwari A, Stalin S, Singh S, Shukla PK. Deep transfer learning based classification model for COVID-19 disease. Ing Rech Biomed. (2020) 1-6. doi: 10.1016/j.irbm.2020.05.003

42. Peng Y, Tang YX, Lee S, Zhu Y, Summers RM, Lu Z. COVID19-CT-CXR: a freely accessible and weakly labeled chest X-ray and CT image collection on COVID-19 from biomedical literature. ArXiv. (2020). doi: 10.1109/TBDATA.2020.3035935

43. Pu J, Leader J, Bandos A, Shi J, Du P, Yu J, et al. Any unique image biomarkers associated with COVID-19? Eur Radiol. (2020) 30:1-7. doi: 10.1007/s00330-020-06956-w

44. Raajan NR, Lakshmi VSR, Prabaharan N. Non-invasive technique-based novel corona (COVID-19) virus detection using CNN. Natl Acad Sci Lett. (2020) 1-4. doi: 10.1007/s40009-020-01009-8

45. Rajaraman S, Siegelman J, Alderson PO, Folio LS, Folio LR, Antani SK. Iteratively pruned deep learning ensembles for COVID-19 detection in chest X-rays. IEEE Access. (2020) 8:115041-50. doi: 10.1109/ACCESS.2020.3003810

46. Sakagianni A, Feretzakis G, Kalles D, Koufopoulou C, Kaldis V. Setting up an easy-to-use machine learning pipeline for medical decision support: a case study for COVID-19 diagnosis based on deep learning with CT scans. Stud Health Technol Inform. (2020) 272:13-6. doi: 10.3233/SHTI200481

47. Sharma S. Drawing insights from COVID-19-infected patients using CT scan images and machine learning techniques: a study on 200 patients. Environ Sci Pollut Res Int. (2020) 27:1-9. doi: 10.21203/rs.3.rs-23863/v1

48. Singh D, Kumar V, Vaishali, Kaur M. Classification of COVID-19 patients from chest CT images using multi-objective differential evolution-based convolutional neural networks. Eur J Clin Microbiol Infect Dis. (2020) 39:1379-89. doi: 10.1007/s10096-020-03901-Z

49. Song J, Wang H, Liu Y, Wu W, Dai G, Wu Z, et al. End-to-end automatic differentiation of the coronavirus disease 2019 (COVID-19) from viral pneumonia based on chest CT. Eur J Nucl Med Mol Imaging. (2020) 47:1-9. doi: 10.1007/s00259-020-04929-1

50. Wang J, Bao Y, Wen Y, Lu H, Luo H, Xiang Y, et al. Priorattention residual learning for more discriminative COVID-19 screening in CT images. IEEE Trans Med Imaging. (2020) 39:2572-83. doi: 10.1109/TMI.2020.2994908

51. Wang S, Zha Y, Li W, Wu Q, Li X, Niu M, et al. A fully automatic deep learning system for COVID-19 diagnostic and prognostic analysis. Eur Respir J. (2020) 56:2000775. doi: 10.1183/13993003.00775-2020

52. Warman A, Warman P, Sharma A, Parikh P, Warman R, Viswanadhan $\mathrm{N}$, et al. Interpretable artificial intelligence for COVID-19 diagnosis from chest CT reveals specificity of ground-glass opacities. medRxiv. (2020) 1-13. doi: 10.1101/2020.05.16.20103408

53. Wu X, Hui H, Niu M, Li L, Wang L, He B, et al. Deep learning-based multi-view fusion model for screening 2019 novel coronavirus pneumonia: a multicentre study. Eur J Radiol. (2020) 128:109041. doi: 10.1016/j.ejrad.2020.109041

54. Xu X, Jiang X, Ma C, Du P, Li X, Lv S, et al. A deep learning system to screen novel coronavirus disease 2019 pneumonia. Engineering. (2020) 6:1-7. doi: 10.1016/j.eng.2020.04.010

55. Xu Y, Ma L, Yang F, Chen Y, Ma K, Yang J, et al. A collaborative online AI engine for CT-based COVID-19 diagnosis. medRxiv. (2020). doi: $10.1101 / 2020.05 .10 .20096073$

56. Yan $\mathrm{T}$, Wong $\mathrm{PK}$, Ren $\mathrm{H}$, Wang $\mathrm{H}$, Wang J, Li Y. Automatic distinction between COVID-19 and common pneumonia using multi-scale convolutional neural network on chest CT scans. Chaos Solitons Fractals. (2020) 140:110153. doi: 10.1016/j.chaos.2020.110153
57. Yang S, Jiang L, Cao Z, Wang L, Cao J, Feng R, et al. Deep learning for detecting corona virus disease 2019 (COVID-19) on highresolution computed tomography: a pilot study. Ann Transl Med. (2020) 8:450. doi: $10.21037 /$ atm.2020.03.132

58. Yu Z, Li X, Sun H, Wang J, Zhao T, Chen H, et al. Rapid identification of COVID-19 severity in CT scans through classification of deep features. Biomed Eng Online. (2020) 19:63. doi: 10.1186/s12938-020-00807-x

59. Al-Karawi D, Al-Zaidi S, Polus N, Jassim S. Machine learning analysis of chest CT scan images as a complementary digital test of coronavirus (COVID-19) patients. medRxiv. (2020) 1-8. doi: $10.1101 / 2020.04 .13 .20063479$

60. Alom MZ, Rahman M, Nasrin MS, Taha TM, Asari VK. COVID_MTNet: COVID-19 detection with multi-task deep learning approaches. arXiv. (2020) Preprint arXiv:200403747.

61. Barstugan M, Ozkaya U, Ozturk S. Coronavirus (covid-19) classification using ct images by machine learning methods. arXiv. (2020) Preprint arXiv:200309424.

62. Chen J, Wu L, Zhang J, Zhang L, Gong D, Zhao Y, et al. Deep learning-based model for detecting 2019 novel coronavirus pneumonia on high-resolution computed tomography. Sci Rep. (2020) 10:1-11. doi: 10.1101/2020.02.25.20021568

63. Farid AA, Selim GI, Awad H, Khater A. A novel approach of CT images feature analysis and prediction to screen for corona virus disease (COVID19). Int J Sci Eng Res. (2020) 11:1-9. doi: 10.14299/ijser.2020.03.02

64. Gozes O, Frid-Adar M, Greenspan H, Browning PD, Zhang H, Ji W, et al. Rapid ai development cycle for the coronavirus (covid-19) pandemic: initial results for automated detection \& patient monitoring using deep learning CT image analysis. arXiv. (2020) Preprint arXiv:200305037.

65. Jin C, Chen W, Cao Y, Xu Z, Zhang X, Deng L, et al. Development and evaluation of an AI system for COVID-19 diagnosis. medRxiv. (2020) 11:1-14. doi: 10.1101/2020.03.20.20039834

66. Jin S, Wang B, Xu H, Luo C, Wei L, Zhao W, et al. AI-assisted CT imaging analysis for COVID-19 screening: building and deploying a medical AI system in four weeks. medRxiv. (2020). doi: 10.1101/2020.03.19.20039354

67. Kassani SH, Kassasni PH, Wesolowski MJ, Schneider KA, Deters R. Automatic detection of coronavirus disease (COVID-19) in X-ray and CT images: a machine learning-based approach. arXiv. (2020) Preprint arXiv:200410641.

68. Ozkaya U, Ozturk S, Barstugan M. Coronavirus (COVID-19) classification using deep features fusion and ranking technique. arXiv. (2020) Preprint arXiv:200403698. doi: 10.1007/978-3-030-55258-9_17

69. Shi F, Xia L, Shan F, Wu D, Wei Y, Yuan H, et al. Large-scale screening of covid-19 from community acquired pneumonia using infection size-aware classification. arXiv. (2020) Preprint arXiv:200309860. doi: 10.1088/1361-6560/abe838

70. Zheng C, Deng X, Fu Q, Zhou Q, Feng J, Ma H, et al. Deep learning-based detection for COVID-19 from chest CT using weak label. medRxiv. (2020) 1-13. doi: 10.1101/2020.03.12.20027185

71. Apostolopoulos ID, Mpesiana TA. Covid-19: automatic detection from X-ray images utilizing transfer learning with convolutional neural networks. Phys Eng Sci Med. (2020) 43:635-40. doi: 10.1007/s13246-020-00865-4

72. Brunese L, Mercaldo F, Reginelli A, Santone A. Explainable deep learning for pulmonary disease and coronavirus COVID-19 detection from X-rays. Comput Methods Programs Biomed. (2020) 196:105608. doi: 10.1016/j.cmpb.2020.105608

73. Chowdhury MEH, Rahman T, Khandakar A, Mazhar R, Kadir MA, Mahbub ZB, et al. Can AI help in screening viral and COVID-19 pneumonia? IEEE Access. (2020) 8:132665-76. doi: 10.1109/ACCESS.2020.3010287

74. Civit-Masot J, Luna-Perejón F, Morales MD, Civit A. Deep learning system for COVID-19 diagnosis aid using X-ray pulmonary images. Appl Sci. (2020) 10:4640. doi: 10.3390/app10134640

75. Elaziz MA, Hosny KM, Salah A, Darwish MM, Lu S, Sahlol AT. New machine learning method for image-based diagnosis of COVID-19. PLoS ONE. (2020) 15:e0235187. doi: 10.1371/journal.pone.0235187

76. Hassantabar S, Ahmadi M, Sharifi A. Diagnosis and detection of infected tissue of COVID-19 patients based on lung x-ray image using 
convolutional neural network approaches. Chaos Solitons Fractals. (2020) 140:110170. doi: 10.1016/j.chaos.2020.110170

77. Islam MZ, Islam MM, Asraf A. A combined deep CNN-LSTM network for the detection of novel coronavirus (COVID-19) using X-ray images. Inform Med Unlocked. (2020) 20:100412. doi: 10.1016/j.imu.2020.100412

78. Khan AI, Shah JL, Bhat MM. CoroNet: a deep neural network for detection and diagnosis of COVID-19 from chest $\mathrm{x}$-ray images. Comput Methods Programs Biomed. (2020) 196:105581. doi: $10.1016 /$ j.cmpb.2020.105581

79. Khuzani AZ, Heidari M, Shariati SA. COVID-Classifier: an automated machine learning model to assist in the diagnosis of COVID-19 infection in chest x-ray images. medRxiv. (2020).

80. Ko H, Chung H, Kang WS, Kim KW, Shin Y, Kang SJ, et al. COVID-19 pneumonia diagnosis using a simple $2 \mathrm{D}$ deep learning framework with a single chest CT image: model development and validation. J Med Internet Res. (2020) 22:e19569. doi: 10.2196/19569

81. Loey M, Smarandache F, Khalifa NEM. Within the lack of chest COVID19 X-ray dataset: a novel detection model based on GAN and deep transfer learning. Symmetry. (2020) 12:651. doi: 10.3390/sym12040651

82. Mahmud T, Rahman MA, Fattah SA. CovXNet: a multi-dilation convolutional neural network for automatic COVID-19 and other pneumonia detection from chest X-ray images with transferable multi-receptive feature optimization. Comput Biol Med. (2020) 122:103869. doi: 10.1016/j.compbiomed.2020.103869

83. Martínez F, Martínez F, Jacinto E. Performance evaluation of the NASnet convolutional network in the automatic identification of COVID-19. Int J Adv Sci Engin Information Technol. (2020) 10:662-7. doi: 10.18517/ijaseit.10.2.11446

84. Minaee S, Kafieh R, Sonka M, Yazdani S, Jamalipour Soufi G. Deep-COVID: predicting COVID-19 from chest X-ray images using deep transfer learning. Med Image Anal. (2020) 65:101794. doi: 10.1016/j.media.2020.101794

85. Narayan Das N, Kumar N, Kaur M, Kumar V, Singh D. Automated deep transfer learning-based approach for detection of COVID19 infection in chest X-rays. Ing Rech Biomed. (2020) 1-7. doi: 10.1016/j.irbm.2020.07.001

86. Nour M, Cömert Z, Polat K. A novel medical diagnosis model for COVID-19 infection detection based on deep features and Bayesian optimization. Appl Soft Comput. (2020) 97:1-14. doi: 10.1016/j.asoc.2020.106580

87. Novitasari DCR, Hendradi R, Caraka RE, Rachmawati Y, Fanani NZ, Syarifudin A, et al. Detection of COVID-19 chest $\mathrm{x}$-ray using support vector machine and convolutional neural network. Commun Math Biol Neurosci. (2020) 2020:1-19. doi: $10.28919 / \mathrm{cmbn} / 4765$

88. Oh Y, Park S, Ye JC. Deep Learning COVID-19 Features on CXR using limited training data sets. IEEE Trans Med Imaging. (2020) 39:2688-700. doi: 10.1109/TMI.2020.2993291

89. Ozturk T, Talo M, Yildirim EA, Baloglu UB, Yildirim O, Rajendra Acharya U. Automated detection of COVID-19 cases using deep neural networks with X-ray images. Comput Biol Med. (2020) 121:103792. doi: 10.1016/j.compbiomed.2020.103792

90. Pandit MK, Banday SA. SARS n-CoV2-19 detection from chest $\mathrm{x}$-ray images using deep neural networks. Int J Pervasive Comput Commun. (2020) 16:19. doi: 10.1108/IJPCC-06-2020-0060

91. Pereira RM, Bertolini D, Teixeira LO, Silla CN, Jr., Costa YMG. COVID-19 identification in chest X-ray images on flat and hierarchical classification scenarios. Comput Methods Programs Biomed. (2020) 194:105532. doi: 10.1016/j.cmpb.2020.105532

92. Rahaman MM, Li C, Yao Y, Kulwa F, Rahman MA, Wang Q, et al. Identification of COVID-19 samples from chest X-Ray images using deep learning: a comparison of transfer learning approaches. J Xray Sci Technol. (2020) 28:1-19. doi: 10.3233/XST-200715

93. Rahimzadeh M, Attar A. A modified deep convolutional neural network for detecting COVID-19 and pneumonia from chest X-ray images based on the concatenation of Xception and ResNet50V2. Inform Med Unlocked. (2020) 19:100360. doi: 10.1016/j.imu.2020.100360
94. Sethy PK, Behera SK, Ratha PK, Biswas P. Detection of coronavirus disease (COVID-19) based on deep features and support vector machine. Int $J$ Math Eng Manage Sci. (2020) 5:643-51. doi: 10.33889/IJMEMS.2020.5.4.052

95. Shibly KH, Dey SK, Islam MT, Rahman MM. COVID faster R$\mathrm{CNN}$ : a novel framework to diagnose novel coronavirus disease (COVID-19) in X-ray images. Inform Med Unlocked. (2020) 20:100405. doi: 10.1016/j.imu.2020.100405

96. Togaçar M, Ergen B, Cömert Z. COVID-19 detection using deep learning models to exploit social mimic optimization and structured chest X-ray images using fuzzy color and stacking approaches. Comput Biol Med. (2020) 121:103805. doi: 10.1016/j.compbiomed.2020.103805

97. Toraman S, Alakus TB, Turkoglu I. Convolutional capsnet: a novel artificial neural network approach to detect COVID-19 disease from X-ray images using capsule networks. Chaos Solitons Fractals. (2020) 140:110122. doi: 10.1016/j.chaos.2020.110122

98. Tsiknakis N, Trivizakis E, Vassalou EE, Papadakis GZ, Spandidos DA, Tsatsakis A, et al. Interpretable artificial intelligence framework for COVID-19 screening on chest X-rays. Exp Ther Med. (2020) 20:727-35. doi: 10.3892/etm.2020.8797

99. Tuncer T, Dogan S, Ozyurt F. An automated residual exemplar local binary pattern and iterative ReliefF based COVID-19 detection method using chest X-ray image. Chemometr Intell Lab Syst. (2020) 203:104054. doi: 10.1016/j.chemolab.2020.104054

100. Ucar F, Korkmaz D. COVIDiagnosis-Net: deep Bayes-SqueezeNet based diagnosis of the coronavirus disease 2019 (COVID-19) from X-ray images. Med Hypotheses. (2020) 140:109761. doi: 10.1016/j.mehy.2020.109761

101. Vaid S, Kalantar R, Bhandari M. Deep learning COVID-19 detection bias: accuracy through artificial intelligence. Int Orthop. (2020) 44:1539-42. doi: 10.1007/s00264-020-04609-7

102. Waheed A, Goyal M, Gupta D, Khanna A, Al-Turjman F, Pinheiro PR. CovidGAN: data augmentation using auxiliary classifier GAN for improved Covid-19 detection. IEEE Access. (2020) 8:91916-23. doi: 10.1109/ACCESS.2020.2994762

103. Yildirim M, Cinar A. A deep learning based hybrid approach for covid-19 disease detections. Traitement Signal. (2020) 37:461-8. doi: 10.18280/ts.370313

104. Yoo SH, Geng H, Chiu TL, Yu SK, Cho DC, Heo J, et al. Deep learning-based decision-tree classifier for COVID-19 diagnosis from chest X-ray imaging. Front Med. (2020) 7:427. doi: 10.3389/fmed.2020.00427

105. Ghoshal B, Tucker A. Estimating uncertainty and interpretability in deep learning for coronavirus (COVID-19) detection. arXiv. (2020) Preprint arXiv:200310769.

106. Hall LO, Paul R, Goldgof DB, Goldgof GM. Finding covid-19 from chest x-rays using deep learning on a small dataset. arXiv. (2020) 40:114. doi: $10.36227 /$ techrxiv. 12083964

107. Hammoudi K, Benhabiles H, Melkemi M, Dornaika F, Arganda-Carreras I, Collard D, et al. Deep learning on chest X-ray images to detect and evaluate pneumonia cases at the Era of COVID-19. arXiv. (2020) Preprint arXiv:200403399.

108. Hemdan EE-D, Shouman MA, Karar ME. Covidx-net: a framework of deep learning classifiers to diagnose covid-19 in X-ray images. arXiv. (2020) Preprint arXiv:200311055.

109. Jain G, Mittal D, Thakur D, Mittal MK. A deep learning approach to detect Covid-19 coronavirus with X-Ray images. Biocybernet Biomed Eng. (2020). doi: 10.1016/j.bbe.2020.08.008

110. Luz E, Silva PL, Silva R, Moreira G. Towards an efficient deep learning model for covid-19 patterns detection in X-ray images. arXiv. (2020) 31:1-10.

111. Ozturk S, Ozkaya U, Barstugan M. Classification of coronavirus images using shrunken features. medRxiv. (2020). doi: 10.1101/2020.04.03.20048868

112. Zhang J, Xie Y, Li Y, Shen C, Xia Y. Covid-19 screening on chest Xray images using deep learning based anomaly detection. arXiv. (2020) Preprint arXiv:200312338.

113. Ravishankar H, Sudhakar P, Venkataramani R, Thiruvenkadam S, Annangi $\mathrm{P}$, Babu N, et al. Understanding the mechanisms of deep transfer learning for medical images. In: Deep Learning and Data Labeling for Medical Applications: Springer (2016). p. 188-96. 
114. Hosny KM, Kassem MA, Foaud MM. Classification of skin lesions using transfer learning and augmentation with Alexnet. PLoS ONE. (2019) 14:e0217293. doi: 10.1371/journal.pone. 0217293

115. Khan S, Islam N, Jan Z, Din IU, Rodrigues JJC. A novel deep learning based framework for the detection and classification of breast cancer using transfer learning. Pattern Recogn Lett. (2019) 125:1-6. doi: 10.1016/j.patrec.2019. 03.022

116. Singh A, Sengupta S, Lakshminarayanan V. Explainable deep learning models in medical image analysis. J Imaging. (2020) 6:52. doi: 10.3390/jimaging6060052
Conflict of Interest: The authors declare that the research was conducted in the absence of any commercial or financial relationships that could be construed as a potential conflict of interest.

Copyright (c) 2021 Mohammad-Rahimi, Nadimi, Ghalyanchi-Langeroudi, Taheri and Ghafouri-Fard. This is an open-access article distributed under the terms of the Creative Commons Attribution License (CC BY). The use, distribution or reproduction in other forums is permitted, provided the original author(s) and the copyright owner(s) are credited and that the original publication in this journal is cited, in accordance with accepted academic practice. No use, distribution or reproduction is permitted which does not comply with these terms. 